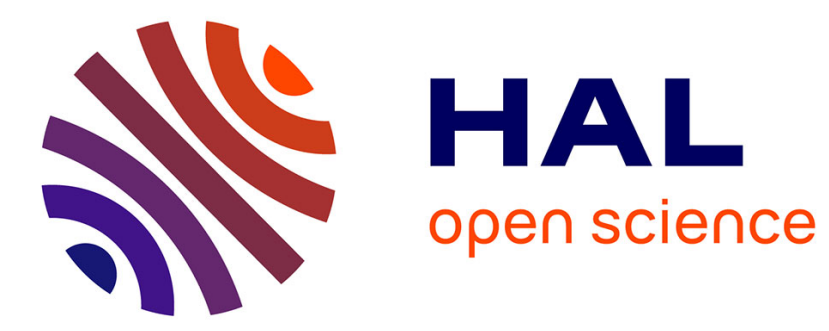

\title{
Local null controllability of a model system for strong interaction between internal solitary waves
}

\author{
Jon Asier Bárcena-Petisco, Sergio Guerrero, Ademir F. Pazoto
}

\section{To cite this version:}

Jon Asier Bárcena-Petisco, Sergio Guerrero, Ademir F. Pazoto. Local null controllability of a model system for strong interaction between internal solitary waves. 2021. hal-02376059v3

\section{HAL Id: hal-02376059 \\ https://hal.science/hal-02376059v3}

Preprint submitted on 20 Mar 2021

HAL is a multi-disciplinary open access archive for the deposit and dissemination of scientific research documents, whether they are published or not. The documents may come from teaching and research institutions in France or abroad, or from public or private research centers.
L'archive ouverte pluridisciplinaire HAL, est destinée au dépôt et à la diffusion de documents scientifiques de niveau recherche, publiés ou non, émanant des établissements d'enseignement et de recherche français ou étrangers, des laboratoires publics ou privés. 


\title{
Local null controllability of a model system for strong interaction between internal solitary waves
}

\author{
Jon Asier Bárcena-Petisco*† ${ }^{*} \quad$ Sergio Guerrero $\$$ Ademir F. Pazoto $\mathbb{I}$
}

\begin{abstract}
In this paper we prove the local null controllability property for a nonlinear coupled system of two Korteweg-de Vries equations posed on a bounded interval and with a source term decaying exponentially on $t=T$. The system was introduced by Gear and Grimshaw to model the interactions of two dimensional, long, internal gravity waves propagation in a stratified fluid. We address the controllability problem by means of a control supported on an interior open subset of the domain and acting on one equation only. The proof consists mainly on proving the controllability of the linearized system, which is done by getting a Carleman estimate for the adjoint system. While doing the Carleman we improve the techniques for dealing with the fact that the solutions of dispersive and parabolic equations with a source term in $\mathbf{L}^{2}$ have a limited regularity. A local inversion theorem is applied to get the result for the nonlinear system.
\end{abstract}

Key words: Carleman inequalities, internal control, Korteweg-de Vries systems, null controllability AMS subject classification: 35Q53, 93B05, 93C20

Abbreviated title: Local null controllability of a system for solitary waves

Acknowledgements: This project has received funding from the European Research Council (ERC) under the European Union's Horizon 2020 research and innovation programme (grant agreement No 694126-DyCon). The first author has been supported by grants from Région Ile-de-France. The first and second authors have been supported by the Agence Nationale de la Recherche (France) research project IFSMACS(ANR-15-CE40-0010). The third author was supported by Conselho Nacional de Desenvolvimento Científico e Tecnológico (Brazil) and Coordenação de Aperfeiçoamento de Pessoal de Nível Superior through Program Print-Capes (Brazil).

\footnotetext{
*Sorbonne Université, Laboratoire Jacques-Louis Lions, F-75005, Paris, France

${ }^{\dagger}$ Present address: [1] Departamento de Matemáticas, Universidad Autónoma de Madrid, 28049 Madrid, Spain.

[2] Chair of Computational Mathematics, Fundación Deusto, Avenida de las Universidades 24, 48007 Bilbao,

Basque Country, Spain; jon.barcena@uam.es; Orcid: 0000-0002-6583-866X

${ }^{\ddagger}$ Corresponding author

${ }^{\S}$ Sorbonne Université, Laboratoire Jacques-Louis Lions, F-75005, Paris, France; guerrero@ann.jussieu.fr

${ }^{I}$ Instituto de Matemática, Universidade Federal do Rio de Janeiro, C.P. 68530 - CEP 21941-909, Rio de Janeiro (RJ), Brazil; ademir@im.ufrj.br
} 


\section{Introduction}

Nonlinear dispersive partial differential equations naturally appear as models describing wave phenomena in various branches of physics and engineering, such as quantum mechanics, nonlinear optics, plasma physics, water waves, and atmospheric sciences. They have received wide attention from the applied science community due to their importance in applications and have also been studied extensively from the theoretical point of view, providing a framework for development of analytical ideas and tools.

In this context, the interaction of internal gravity waves propagating in one horizontal direction has been considered by Gear and Grimshaw [26]. More precisely, the authors derived a model to describe strong interactions of two long internal gravity waves in a stratified fluid, where the two waves are assumed to correspond to different modes of the linearized equations of motion. It can be written as

$$
\begin{aligned}
& u_{t}+u u_{x}+u_{x x x}+a v_{x x x}+k_{1} v v_{x}+k_{2}(u v)_{x}=0, \\
& c v_{t}+r v_{x}+v v_{x}+a b u_{x x x}+v_{x x x}+k_{2} b u u_{x}+k_{1} b(u v)_{x}=0,
\end{aligned}
$$

where $a, b, c, r, k_{1}, k_{2}$ are real parameters, the unknowns $u$ and $v$ are real valued functions of the variables $x$ and $t$ and subscripts indicate partial differentiation. Observe that (1.1) has the structure of a pair of Korteweg-de Vries (KdV) equations with both linear and nonlinear coupling terms.

We also refer to [6] for an extensive discussion on the physical relevance of the system in its full structure.

\subsection{Setting of the problem}

In this paper, we are concerned with the study of the controllability properties of the system (1.1), posed on a bounded interval $(0, L)$, with homogeneous boundary conditions. The main purpose is to steer the solutions of the system to the rest by means of a control supported on an interior open subset $\omega$ of $(0, L)$, acting on one equation only. As usual, we denote

$$
\begin{aligned}
& Q:=(0, T) \times(0, L), \quad Q_{\omega}:=(0, T) \times \omega, \text { for any open subset } \omega \text { of }(0, L), \\
& 1_{\omega}, \text { the characteristic function on } \omega .
\end{aligned}
$$

With the notation above, our attention is given to the following distributed control system 
with initial data $\left(u^{0}, v^{0}\right)$, source term $\left(\mathfrak{h}_{1}, \mathfrak{h}_{2}\right)$ and control $f$ :

$$
\begin{cases}u_{t}+u u_{x}+u_{x x x}+a v_{x x x}+k_{1} v v_{x}+k_{2}(u v)_{x}=\mathfrak{h}_{1}, & \text { in } Q, \\ c v_{t}+r v_{x}+v v_{x}+a b u_{x x x}+v_{x x x}+k_{2} b u u_{x}+k_{1} b(u v)_{x}=\mathfrak{h}_{2}+f 1_{\omega}, & \text { in } Q, \\ u(\cdot, 0)=u(\cdot, L)=u_{x}(\cdot, L)=0, & \text { on }(0, T), \\ v(\cdot, 0)=v(\cdot, L)=v_{x}(\cdot, L)=0, & \text { on }(0, T), \\ u(0, \cdot)=u^{0}, \quad v(0, \cdot)=v^{0}, & \text { on }(0, L) .\end{cases}
$$

Under these conditions, considerations will be given to the following null controllability problem:

Given $T>0$, an initial state $\left(u^{0}, v^{0}\right)$ in a certain space, and a source term $\left(\mathfrak{h}_{1}, \mathfrak{h}_{2}\right)$ in a certain space, can one find an appropriate control $f$, so that the system (1.3) admits a solution $(u, v)$ which satisfies $(u(T, \cdot), v(T, \cdot))=(0,0)$ ?

Our main result gives a positive answer to this question. The proof combines the analysis of the linearized system and an inverse function theorem for the full system. In order to analyze the linearized system, we follow the classical duality approach [21, 32], which reduces the null controllability property to the proof of an observability inequality for the solutions of the corresponding adjoint system. Here, this is done by using Carleman estimates.

In order to overcome some technical difficulties that will become clear later (see fourth comment in Section 4.3), we assume that the coefficients $a, b$ and $c$ satisfy:

$$
a \neq 0, \quad b, c>0 \quad \text { and } \quad a^{2} b<1 .
$$

To our knowledge, this problem has not been addressed in the literature and the existing developments do not allow to give an immediate answer to it. Indeed, the controllability problem for system 1.1] was first solved when the control acts through the boundary conditions [8, 9, 17, 35. The results are obtained combining the analysis of the linearized system and the Banach's fixed-point theorem. In order to study the linear problem, the authors follow closely the ideas introduced by Rosier [36] while studying the boundary controllability of the KdV equation (based on compactness arguments, a spectral analysis of the state operator and multipliers). But, being different from other systems, the length $L$ of the spatial domain may play a crucial role in determining the controllability of the system, specially when some configurations of the controls input are allowed to be used. This phenomenon, the so-called critical length phenomenon, was observed for the first time by Rosier in [36]. Roughly speaking, it was proved the existence of a finite dimensional subspace $M$ of $L^{2}(0, L)$, which is not reachable by the $\mathrm{KdV}$ equation, when starting from the origin, if $L$ belongs to a countable set of lengths. 
More recently, by using the approach used in the present paper, the internal null controllability for the Hirota-Satsuma system was addressed in [16]. However, due to the complexity of the system (1.1), which has coupling constituted by third order terms, the control problem presents new difficulties we have to deal with. Then, we develop techniques applicable to this more complicated situation, which allow to slightly improve the result present in [16] and, in addition, can also be applied to other dispersive and parabolic systems.

To conclude, we can mention the work [10] where the authors consider the problem of controlling pointwise, by means of a time dependent Dirac measure supported at a given point, the linear system associated with (1.1) on the unit circle. The results are obtained by means of spectral analysis and Fourier expansion of the solutions.

\subsection{Main results}

Before stating our main result, we introduce the space

$$
H^{p, p^{\prime}}(Q):=H^{p}\left(0, T ; L^{2}(0, L)\right) \cap L^{2}\left(0, T ; H^{p^{\prime}}(0, L)\right), \quad \text { for } p, p^{\prime} \geq 0,
$$

endowed with its natural norm, where $Q$ was defined in $(1.2)$. In addition, to shorten the notation we denote

- $U:=(u, v), U^{0}:=\left(u^{0}, v^{0}\right)$,

- $\mathbf{V}=V^{2}$, for $V$ any Banach space (like $L^{2}, H_{0}^{1}$ or $H^{p, p^{\prime}}$ ),

- $\Phi:=(\varphi, \phi)$ the adjoint variable,

- $C$ (resp. q) a generic positive constant (resp. polynomial of positive coefficients) that may be different each time.

The main result that we prove in this paper concerns the local null controllability of 1.3 :

Theorem 1.1. Let $L>0, T>0$ and assume that (1.4) is satisfied. Then, there is $C>0$ such that for all $\mathfrak{h}_{1}, \mathfrak{h}_{2}$ satisfying

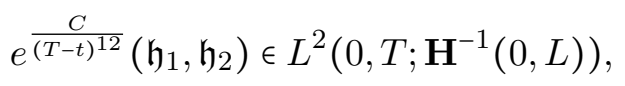

there is $\delta>0$ such that for all $\left\|U^{0}\right\|<\delta$ there is $f \in L^{2}\left(Q_{\omega}\right)$ and $U \in C\left([0, T] ; \mathbf{L}^{2}(0, L)\right)$ satisfying that $(U, f)$ is a solution of $(1.3)$ and $U(T, \cdot)=0$. 
As usual, we obtain the null controllability of $(1.3)$ by proving the null controllability of the linearized system around 0 . That system is given by

$$
\begin{cases}\mathcal{L} U=\left(h_{1}, h_{2}+f 1_{\omega}\right), & \text { in } Q, \\ U(\cdot, 0)=U(\cdot, L)=U_{x}(\cdot, L)=0, & \text { on }(0, T), \\ U(0, \cdot)=U^{0}, & \text { on }(0, L),\end{cases}
$$

for $h_{1}, h_{2} \in L^{2}\left(0, T ; \mathbf{H}^{-1}(0, L)\right)$ decaying exponentially at $t=T$ as specified in Section 4.1 , $U^{0} \in \mathbf{L}^{2}(0, L)$ and

$$
\mathcal{L} U:=\left(u_{t}+u_{x x x}+a v_{x x x}, c v_{t}+r v_{x}+a b u_{x x x}+v_{x x x}\right) .
$$

First, in Section 2 we study the main properties of the associated Cauchy problem, which is given by

$$
\begin{cases}\mathcal{L} U=G, & \text { in } Q, \\ U(\cdot, 0)=U(\cdot, L)=U_{x}(\cdot, L)=0, & \text { on }(0, T), \\ U(0, \cdot)=U^{0}, & \text { on }(0, L),\end{cases}
$$

for $U^{0} \in \mathbf{L}^{2}(0, L)$ and $G \in L^{2}\left(0, T ; \mathbf{H}^{-1}(0, L)\right)$. Afterwards, in order to show the null controllability of (1.5) we follow the classical duality approach [21, 32, which reduces the problem to prove an observability inequality for the solutions of the corresponding adjoint system, which is given by

$$
\begin{cases}\mathcal{L}^{*} \Phi=G, & \text { in } Q, \\ \Phi(\cdot, 0)=\Phi(\cdot, L)=\Phi_{x}(\cdot, 0)=0, & \text { on }(0, T), \\ \Phi(T, \cdot)=\Phi^{T}, & \text { on }(0, L),\end{cases}
$$

for $\Phi^{T} \in \mathbf{L}^{2}(0, L), G \in \mathbf{L}^{2}(Q)$ and

$$
\mathcal{L}^{*} \Phi:=\left(-\varphi_{t}-\varphi_{x x x}-a b \phi_{x x x},-c \phi_{t}-r \phi_{x}-a \varphi_{x x x}-\phi_{x x x}\right) .
$$

In fact, we study the main properties of 1.8 in Section 2 , and then we prove a Carleman estimate in Section 3 ,

One of the main contributions of this paper is to make it clear the difficulty of getting Carleman estimates for any well-posed dispersive or parabolic system with a force $G \in \mathbf{L}^{2}(Q)$ or $G=0$. Indeed, to overcome the fact that solution $\Phi$ is not regular enough (because $G \in \mathbf{L}^{2}(Q)$ ) the proof of the Carleman estimate presents the novelty that we decompose $\Phi$ as the sum of the solution of three Cauchy problems. In the literature, the adjoint variable has been decomposed as the sum of the solution of two Cauchy problems (see, for instance, [13]), but for (1.8) we 
clearly need more. This implies some changes in the proof with respect to the existing results, which are explained in Section 3.2 and can be applied for many other dispersive and parabolic systems.

\subsection{Historical background}

There is a huge literature on controllability results with controls having a reduced number of components concerning parabolic equations (see the survey [4] and the references therein). For instance, there are a lot of results about Navier-Stokes-like systems, such as [7, 13, 14, 18, [19, 24, 30, 33]. It has also been studied, for example, the controllability in cascade-like systems [29, 15, the null controllability in the context of linear thermoelasticity [31], the controllability to trajectories in phase-field models [3], the existence of insensitizing controls for the heat equation [20], and the controllability in reaction-diffusion systems [2]. However, as for KdV-like systems, it is a very recent research topic which has been studied only in [10] and [16].

The main difference of the problem we consider in this paper with respect to the above cited papers, except [10], is the coupling. Indeed, in the papers cited above (and in the literature, as far as we know) the coupling is constituted by a zero, first or second order term. However, in this paper the coupling is constituted by a third order term. In this sense, the main contribution of this paper is in the technicalities of the Carleman, which provides a technique to move from the controllability of the dipersive linearized system with null force to the controllability of the linearized system with a force decaying in $T$ (usually a necessary step to prove the local null controllability of some nonlinear systems).

In [10], the authors consider the problem of controlling pointwise, by means of a time dependent Dirac measure supported by a given point, the linearized system on the unit circle. More precisely, by means of spectral analysis and Fourier expansion they prove, under general assumptions on the physical parameters of the system, a pointwise observability inequality which leads to the pointwise controllability by using one control function. It is also possible to find the sharp time of the controllability.

By contrast, the study of the boundary controllability properties is considerably more developed. The first result was obtained in [34, when the model is posed on a periodic domain and $r=0$. In this case, a diagonalization of the main terms allows to decouple the corresponding linear system into two scalar KdV equations and to use the previous results available in the literature. Later on, in [35], the authors proved the local exact boundary controllability property for the nonlinear system (1.1) posed on a bounded interval. Their result was improved in [9], [17] and in [8, where the same boundary control problem was addressed with a different set of boundary conditions. In all those works, except in [17], the results were proved by applying 
the duality approach and some ideas introduced by Rosier in [36]. Then, it was discovered that whether the system is exactly controllable depends on the length of the spatial domain, i.e., if $L$ does not lie in the so-called countable set of critical lengths. More precisely, the observability inequality is proved with the aid of a compactness argument that leads the issue to a nonstandard unique continuation principle for the eigenvalues of the state operator associated with the model. Then, the problem is solved by using the Paley-Wiener method introduced by Rosier, which allows the authors determine explicitly all the critical lengths for which the exact controllability fails for the linearized system. The same problem was addressed in [17] when only two controls act on the Neumann boundary conditions. In this case, the analysis of the linearized system is much more complicated. Therefore, the authors used a direct approach based on the multiplier technique that gives the observability inequality for small values of the length $L$ and large time of control $T$.

Before closing this section, we mention the work [12], in which the boundary controllability problem was addressed for a Boussinesq system of KdV type.

The rest of the paper is organized as follows: in Section 2 we study the regularity estimates of some elliptic and evolution problems, in Section 3 we prove a Carleman inequality for (1.8), and finally in Section 4 we end the proof of Theorem 1.1 .

\section{Some elliptic and evolution problems}

In this section we first prove a Poincaré inequality and secondly we prove some existence, uniqueness and regularity results for (1.7) and 1.8 .

\subsection{A Poincaré inequality}

In this subsection we prove the following Poincaré inequality:

Proposition 2.1. Let $L>0$ and $i \in \mathbb{N}$. Then, there is $C>0$ such that for all $w \in H^{2+i}(0, L)$ satisfying

$$
w(0)=w(L)=w^{\prime}(0)=0,
$$

we have the estimate

$$
\|w\|_{H^{2+i}(0, L)} \leq C\left\|w_{x x x}\right\|_{H^{i-1}(0, L)} .
$$

Remark 2.2. By symmetry, for all $i \in \mathbb{N}$ we also have 2.2 for all $w \in H^{2+i}(0, L)$ satisfying

$$
w(0)=w(L)=w^{\prime}(L)=0 .
$$


Proposition 2.1, for $i \geq 1$, is well-known and has been used in the literature (see for instance [27]). However, as far as we know, there is no reference for $i=0$.

In order to prove Proposition 2.1, we use the following interpolation result:

Lemma 2.3. Let $L>0$. Then, there is $C>0$ such that for all $z \in H^{1}(0, L)$ we have that

$$
\left\|z_{x}\right\|_{L^{2}(0, L)} \leq C\left(\|z\|_{L^{2}(0, L)}+\left\|z_{x x}\right\|_{H^{-1}(0, L)}\right) .
$$

Proof of Lemma 2.3. We fix $\chi \in \mathcal{D}(0, L)$ such that $\int_{0}^{L} \chi=1$. Then, for any $\psi \in \mathcal{D}(0, L)$ we have the estimate below

$$
\begin{array}{r}
\left|\int_{0}^{L} z_{x} \psi\right|=\left|\int_{0}^{L} z_{x}\left(\int_{0}^{x}\left(\psi(s)-\chi(s) \int_{0}^{L} \psi(z) d z\right) d s\right)_{x} d x+\int_{0}^{L} z_{x} \chi \int_{0}^{L} \psi(s) d s d x\right| \\
=\mid\left\langle-z_{x x},\left.\int_{0}^{x}\left(\psi(s)-\chi(s) \int_{0}^{L} \psi(z) d z\right) d s\right|_{H^{-1}(0, L) \times H_{0}^{1}(0, L)}-\int_{0}^{L} z \chi_{x} \int_{0}^{L} \psi(s) d s\right| \\
\leq C\left(\left\|z_{x x}\right\|_{H^{-1}(0, L)}+\|z\|_{L^{2}(0, L)}\right)\|\psi\|_{L^{2}(0, L)} .
\end{array}
$$

Consequently, 2.3. is obtained from Riesz theorem and the density of $\mathcal{D}(0, L)$ in $L^{2}(0, L)$.

Proof of Proposition 2.1. It suffices to prove Proposition 2.1 for $i=0$. Indeed, once that case is proved, we have the following estimate

$$
\begin{aligned}
& \|w\|_{H^{2+i}(0, L)} \leq C\left(\|w\|_{H^{2}(0, L)}+\left\|w_{x x x}\right\|_{H^{i-1}(0, L)}\right) \\
& \quad \leq C\left(\left\|w_{x x x}\right\|_{H^{-1}(0, L)}+\left\|w_{x x x}\right\|_{H^{i-1}(0, L)}\right) \leq C\left\|w_{x x x}\right\|_{H^{i-1}(0, L)} .
\end{aligned}
$$

We prove the case $i=0$ by contradiction.

If Proposition 2.1 is false for $i=0$, there is a sequence $w^{j} \in H^{2}(0, L)$ satisfying (2.1) such that

$$
\left\|w^{j}\right\|_{H^{2}(0, L)}=1
$$

and

$$
\left\|w_{x x x}^{j}\right\|_{H^{-1}(0, L)} \rightarrow 0 .
$$

By compactness, we can suppose that $w^{j}$ is convergent in $H^{7 / 4}(0, L)$ to some function $w$ (see, for instance, [1]). The convergence implies that $w$ satisfies (2.1) and that $w_{x x x}=0$ as a distribution. Hence, we can prove easily that $w=0$. Consequently,

$$
\left\|w^{j}\right\|_{H^{1}(0, L)} \rightarrow 0
$$

which together with Lemma 2.3 for $z=w_{x}$ and $(2.6)$ implies that

$$
\left\|w_{x x}^{j}\right\|_{L^{2}(0, L)} \rightarrow 0 \text {. }
$$

Thus, we find from (2.7) and 2.8) that $\left\|w^{j}\right\|_{H^{2}(0, L)} \rightarrow 0$, contradicting 2.5. 


\subsection{Existence, uniqueness and regularity properties of the solutions of some dissipative evolution problems}

We have the following result for (1.7), which is a consequence of the results presented in [35, Section 2.1] and classical semigroup theory:

Lemma 2.4. Let $L>0, T>0$ and assume that 1.4 is satisfied. Then, for any $U^{0} \in \mathbf{L}^{2}(0, L)$ and $G \in L^{1}\left(0, T ; \mathbf{L}^{2}(0, L)\right)$ system (1.7) admits a unique solution

$$
U \in C\left([0, T] ; \mathbf{L}^{2}(0, L)\right) \cap L^{2}\left(0, T ; \mathbf{H}^{1}(0, L)\right)
$$

Moreover, there is $C>0$ independent of $U^{0}$ and $G$ such that

$$
\|U\|_{C\left([0, T] ; \mathbf{L}^{2}(0, L)\right) \cap L^{2}\left(0, T ; \mathbf{H}^{1}(0, L)\right)} \leq C\left(\left\|U^{0}\right\|_{\mathbf{L}^{2}(0, L)}+\|G\|_{L^{1}\left(0, T ; \mathbf{L}^{2}(0, L)\right)}\right) .
$$

In addition, if $U^{0} \in \mathcal{D}(0, L)$ and $G \in \mathcal{D}(Q)$ we have that $U \in \mathbf{C}^{\infty}(\bar{Q})$.

In a similar way, we have an analogous result for the adjoint system:

Lemma 2.5. Let $L>0, T>0$ and assume that (1.4) is satisfied. Then, for any $\Phi^{T} \in \mathbf{L}^{2}(0, L)$ and $G \in L^{1}\left(0, T ; \mathbf{L}^{2}(0, L)\right)$ system $(1.8)$ admits a unique solution

$$
\Phi \in C\left([0, T] ; \mathbf{L}^{2}(0, L)\right) \cap L^{2}\left(0, T ; \mathbf{H}^{1}(0, L)\right) .
$$

Moreover, there is $C>0$ independent of $\Phi^{T}$ and $G$ such that

$$
\|\Phi\|_{\left.C\left([0, T] ; \mathbf{L}^{2}(0, L)\right)\right) \cap L^{2}\left(0, T ; \mathbf{H}^{1}(0, L)\right)} \leq C\left(\left\|\Phi^{T}\right\|_{\mathbf{L}^{2}(0, L)}+\|G\|_{L^{1}\left(0, T ; \mathbf{L}^{2}(0, L)\right)}\right) .
$$

In addition, if $\Phi^{T} \in \mathcal{D}(0, L)$ and $G \in \mathcal{D}(Q)$ we have that $\Phi \in \mathbf{C}^{\infty}(\bar{Q})$.

To continue with, we prove more accurate results about the regularity of the solutions of (1.8):

Proposition 2.6. Let $L>0$ and assume that (1.4) is satisfied. Then:

1. For all $T>0, \Phi^{T} \in \mathbf{L}^{2}(0, L)$ and $G \in L^{2}\left(0, T ; \mathbf{H}^{-1}(0, L)\right)$ system 1.8 admits a unique solution

$$
\Phi \in C\left([0, T] ; \mathbf{L}^{2}(0, L)\right) \cap L^{2}\left(0, T ; \mathbf{H}^{1}(0, L)\right) .
$$

Moreover, there is $C>0$ independent of $T, \Phi^{T}$ and $G$ such that

$$
\|\Phi\|_{\left.C\left([0, T] ; \mathbf{L}^{2}(0, L)\right)\right) \cap L^{2}\left(0, T ; \mathbf{H}^{1}(0, L)\right)} \leq C(1+T)\left(\left\|\Phi^{T}\right\|_{\mathbf{L}^{2}(0, L)}+\|G\|_{L^{2}\left(0, T ; \mathbf{H}^{-1}(0, L)\right)}\right) .
$$


2. There is $C>0$ such that for all $T>0$ and $G \in \mathbf{L}^{2}(Q)$ we have

$$
\Phi \in L^{2}\left(0, T ; \mathbf{H}^{2}(0, L)\right) \cap H^{1}\left(0, T ; \mathbf{H}^{-1}(0, L)\right)
$$

satisfying the estimate

$$
\|\Phi\|_{L^{2}\left(0, T ; \mathbf{H}^{2}(0, L)\right) \cap H^{1}\left(0, T ; \mathbf{H}^{-1}(0, L)\right)} \leq C(1+T)\|G\|_{\mathbf{L}^{2}(Q)}
$$

for $\Phi$ the energy solution of 1.8 with initial value $\Phi^{T}=0$.

3. There is $C>0$ such that for all $T>0$ and $G \in L^{2}\left(0, T ; \mathbf{H}_{0}^{1}(0, L)\right)$ we have $\Phi \in \mathbf{H}^{1,3}(Q)$ satisfying the estimate

$$
\|\Phi\|_{\mathbf{H}^{1,3}(Q)} \leq C\left(1+T^{3}\right)\|G\|_{L^{2}\left(0, T ; \mathbf{H}_{0}^{1}(0, L)\right)},
$$

for $\Phi$ the energy solution of 1.8 with initial value $\Phi^{T}=0$.

For the proof of Proposition 2.6 we need the following result, which is proved in [27]:

Lemma 2.7. Let $L>0$ and $k>0$. Then, there is $C>0$ such that for all $T>0$ and $g \in L^{2}(Q)$ we have $w \in L^{2}\left(0, T ; H^{2}(0, L)\right)$ satisfying the estimate

$$
\|w\|_{L^{2}\left(0, T ; H^{2}(0, L)\right)} \leq C\|g\|_{L^{2}(Q)}
$$

for $w$ the energy solution of

$$
\begin{cases}-w_{t}-k w_{x x x}=g, & \text { in } Q \\ w(\cdot, 0)=w(\cdot, L)=w_{x}(\cdot, 0)=0, & \text { on }(0, T) \\ w(T, \cdot)=0 & \text { on }(0, L) .\end{cases}
$$

Proof of Proposition 2.6. Step 1: some first facts and presentation of the proof. The uniqueness result of statement 1 is a consequence of the linearity of the problem and of Lemma 2.5 applied to $\Phi^{T}=0$ and $G=0$. Thus, we just have to focus on the existence results and the regularity estimates 2.9)-2.11). For that purpose, thanks to linearity and density results, it actually suffices to prove (2.9)-2.11) when $\Phi^{T} \in \mathcal{D}(0, L)$ (or $\Phi^{T}=0$ ) and $G \in \mathcal{D}(Q)$. We remind the reader that under those hypothesis thanks to Lemma 2.5 the energy solution of 1.8 belongs to $\mathbf{C}^{\infty}(\bar{Q})$. Hence, the rest of the proof is structured as follows: we first diagonalize (1.8) and secondly we prove the estimates 2.9 - 2.11 . 
Step 2: diagonalization of (1.8). We first remark that 1.8 is given by

$$
\begin{cases}-\Phi_{t}^{*}-M \Phi_{x x x}^{*}-\left[\begin{array}{cc}
0 & 0 \\
0 & \frac{r}{c}
\end{array}\right] \Phi_{x}^{*}=\left[\begin{array}{l}
g_{1} \\
\frac{g_{2}}{c}
\end{array}\right], & \text { in } Q, \\
\Phi^{*}(\cdot, 0)=\Phi^{\star}(\cdot, L)=\Phi_{x}^{*}(\cdot, 0)=0, & \text { on }(0, T), \\
\Phi^{*}(T, \cdot)=\left(\Phi^{T}\right)^{*}, & \text { on }(0, L),\end{cases}
$$

for

$$
M:=\left[\begin{array}{cc}
1 & a b \\
\frac{a}{c} & \frac{1}{c}
\end{array}\right], \quad \Phi^{*}:=\left[\begin{array}{c}
\varphi \\
\phi
\end{array}\right], \quad\left(\Phi^{T}\right)^{*}:=\left[\begin{array}{c}
\varphi^{T} \\
\phi^{T}
\end{array}\right] .
$$

We have that the eigenvalues of $M$ are

$$
\lambda_{ \pm}:=\frac{1+\frac{1}{c} \pm \sqrt{\left(1+\frac{1}{c}\right)^{2}-4 \frac{1-a^{2} b}{c}}}{2}=\frac{1+\frac{1}{c} \pm \sqrt{\left(1-\frac{1}{c}\right)^{2}+4 \frac{a^{2} b}{c}}}{2},
$$

which are strictly positive and different by (1.4). Thereby, there is a matrix $P$ such that

$$
P M P^{-1}=\left[\begin{array}{cc}
\lambda_{+} & 0 \\
0 & \lambda_{-}
\end{array}\right] \text {. }
$$

Then, if

$$
\tilde{\Phi}:=P \Phi^{*}
$$

we have that $\tilde{\Phi}$ satisfies the system

$$
\begin{cases}-\tilde{\Phi}_{t}-\left[\begin{array}{cc}
\lambda_{+} & 0 \\
0 & \lambda_{-}
\end{array}\right] \tilde{\Phi}_{x x x}-\tilde{M} \tilde{\Phi}_{x}=\tilde{G}, & \text { in } Q, \\
\tilde{\Phi}(\cdot, 0)=\tilde{\Phi}(\cdot, L)=\tilde{\Phi}_{x}(\cdot, 0)=0, & \text { on }(0, T), \\
\tilde{\Phi}(T, \cdot)=P\left(\Phi^{T}\right)^{*}, & \text { on }(0, L),\end{cases}
$$

for

$$
\tilde{M}:=P\left[\begin{array}{cc}
0 & 0 \\
0 & \frac{r}{c}
\end{array}\right] P^{-1}, \tilde{G}:=P\left[\begin{array}{l}
g_{1} \\
\frac{g_{2}}{c}
\end{array}\right] .
$$

Clearly, it suffices to get the estimates for system (2.16), which is easier because the coupling appears only on the first-order spatial term.

Step 3: proof of 2.9). If we multiply 2.16 by $\tilde{\Phi}$, after integrating by parts and applying Cauchy-Schwarz inequality we obtain

$$
\|\tilde{\Phi}\|_{C\left([0, T] ; \mathbf{L}^{2}(0, L)\right)}^{2} \leq C_{\delta} T\|\tilde{G}\|_{L^{2}\left(0, T ; \mathbf{H}^{-1}(0, L)\right)}^{2}+\frac{\delta}{T}\|\tilde{\Phi}\|_{L^{2}\left(0, T ; \mathbf{H}_{0}^{1}(0, L)\right)}^{2} .
$$

Thus, if we multiply 2.16 by $(L+1-x) \tilde{\Phi}, 2.9$ is obtained by using classical estimates, integration by parts, 2.18 to deal with the first order term and 2.15). 
Step 4: proof of 2.10. We recall that from now on

$$
\tilde{\Phi}^{T}=P\left(\Phi^{T}\right)^{\star}=0 .
$$

Thanks to 2.16) we know that system (1.8) behaves in the higher order terms as two independent $\mathrm{KdV}$ equations. In particular, by considering (2.19), (2.12) ( $g$ is taken as the components of $\left.\tilde{G}+\tilde{M} \tilde{\Phi}_{x}\right)$ and 2.9 we find that

$$
\|\tilde{\Phi}\|_{L^{2}\left(0, T ; \mathbf{H}^{2}(0, L)\right)} \leq C(1+T)\|\tilde{G}\|_{\mathbf{L}^{2}(Q)} .
$$

Hence, we obtain from (2.15), 2.17) and $(2.20)$ that

$$
\|\Phi\|_{L^{2}\left(0, T ; \mathbf{H}^{2}(0, L)\right)} \leq C(1+T)\|G\|_{\mathbf{L}^{2}(Q)} .
$$

We can easily get (2.10) from 2.21) and 1.8 .

Step 5: proof of 2.11). Considering 2.19), we remark that $\Psi:=\tilde{\Phi}_{x x x}$ is the solution of the system

$$
\begin{cases}\Psi_{t}+\left[\begin{array}{cc}
\lambda_{+} & 0 \\
0 & \lambda_{-}
\end{array}\right] \Psi_{x x x}+\tilde{M} \Psi_{x}=\tilde{G}_{x x x}, & \text { in } Q, \\
\Psi(\cdot, 0)=-\hat{M} \tilde{M} \tilde{\Phi}_{x}(\cdot, 0)=0, & \text { on }(0, T), \\
\Psi(\cdot, L)=-\hat{M} \tilde{M} \tilde{\Phi}_{x}(\cdot, L), & \text { on }(0, T), \\
\Psi_{x}(\cdot, 0)=-\hat{M} \tilde{M} \tilde{\Phi}_{x x}(\cdot, 0), & \text { on }(0, T), \\
\Psi(T, \cdot)=0, & \text { on }(0, L),\end{cases}
$$

for

$$
\hat{M}:=\left[\begin{array}{cc}
\lambda_{+}^{-1} & 0 \\
0 & \lambda_{-}^{-1}
\end{array}\right] .
$$

Using that $\tilde{G} \in L^{2}\left(0, T ; \mathbf{H}_{0}^{3}(0, L)\right)$ and considering that the solution can be defined by transposition as in [27, Proposition 1] we find the following estimate:

$$
\|\Psi\|_{\mathbf{L}^{2}(Q)} \leq C(1+T)\left(\|\tilde{G}\|_{L^{2}\left(0, T ; \mathbf{H}_{0}^{1}(0, L)\right)}+\left\|\tilde{\Phi}_{x}(\cdot, L)\right\|_{\mathbf{L}^{2}(0, T)}+\left\|\tilde{\Phi}_{x x}(\cdot, 0)\right\|_{\mathbf{L}^{2}(0, T)}\right) .
$$

Recalling that $\Psi=\tilde{\Phi}_{x x x}$ and 2.20$)$ we obtain from $(2.22)$ that

$$
\|\tilde{\Phi}\|_{L^{2}\left(0, T ; \mathbf{H}^{3}(0, L)\right)} \leq C\left(\left(1+T^{2}\right)\|\tilde{G}\|_{L^{2}\left(0, T ; \mathbf{H}_{0}^{1}(0, L)\right)}+(1+T)\left\|\tilde{\Phi}_{x x}(\cdot, 0)\right\|_{\mathbf{L}^{2}(0, T)}\right) .
$$

Consequently, using the following trace estimate

$$
\left\|\tilde{\Phi}_{x x}(\cdot, 0)\right\|_{\mathbf{L}^{2}(0, T)} \leq \frac{\delta}{1+T}\|\tilde{\Phi}\|_{L^{2}\left(0, T ; \mathbf{H}^{3}(0, L)\right)}+C_{\delta}(1+T)\|\tilde{\Phi}\|_{L^{2}\left(0, T ; \mathbf{H}^{2}(0, L)\right)},
$$

we find from 2.15, 2.17) and 2.23 that

$$
\|\Phi\|_{L^{2}\left(0, T ; \mathbf{H}^{3}(0, L)\right)} \leq C\left(1+T^{3}\right)\|G\|_{L^{2}\left(0, T ; \mathbf{H}_{0}^{1}(0, L)\right)} .
$$

In addition, the $\mathbf{L}^{2}$-norm of $\Phi_{t}$ is estimated from (1.8) and (2.24), and thus we obtain (2.11). 
Two consequences of Proposition 2.6 are the following:

Corollary 2.8. Let $L>0, p \in \mathbb{N}_{*}$ and assume that (1.4) is satisfied. Then, there is a polynomial $q$ such that for all $T>0$ and

$$
G \in H^{p-1}\left(0, T ; \mathbf{H}_{0}^{1}(0, L)\right) \cap L^{2}\left(0, T ; \mathbf{H}^{3 p-3}(0, L)\right)
$$

satisfying

$$
\partial_{t}^{i} G(T, \cdot)=0, \quad \forall i \in\{0,1, \ldots, p-2\},
$$

we have that $\Phi \in \mathbf{H}^{p, 3 p}(Q)$ and the following estimate holds

$$
\|\Phi\|_{\mathbf{H}^{p, 3 p}(Q)} \leq q(T)\left(\|G\|_{H^{p-1}\left(0, T ; \mathbf{H}_{0}^{1}(0, L)\right) \cap L^{2}\left(0, T ; \mathbf{H}^{3 p-3}(0, L)\right)}\right),
$$

for $\Phi$ the energy solution of (1.8) with initial value $\Phi^{T}=0$, and $q$ a polynomial of degree $3 p$.

Corollary 2.9. Let $L>0, p \in \mathbb{N}_{*}$ and assume that (1.4) is satisfied. Then, there a polynomial $q$ such that for all $T>0$ and

$$
G \in H^{p}\left(0, T ; \mathbf{H}^{-1}(0, L)\right) \cap L^{2}\left(0, T ; \mathbf{H}^{3 p-2}(0, L)\right)
$$

satisfying

$$
\partial_{t}^{i} G(T, \cdot)=0, \quad \forall i \in\{0,1, \ldots, p-1\},
$$

we have that $\Phi \in H^{p}\left(0, T ; \mathbf{H}^{1}(0, L)\right) \cap L^{2}\left(0, T ; \mathbf{H}^{3 p+1}(0, L)\right)$ and the following estimate holds

$$
\|\Phi\|_{H^{p}\left(0, T ; \mathbf{H}^{1}(0, L)\right) \cap L^{2}\left(0, T ; \mathbf{H}^{3 p+1}(0, L)\right)} \leq q(T)\|G\|_{H^{p}\left(0, T ; \mathbf{H}^{-1}(0, L)\right) \cap L^{2}\left(0, T ; \mathbf{H}^{3 p-2}(0, L)\right)},
$$

for $\Phi$ the energy solution of (1.8) with initial value $\Phi^{T}=0$, and $q$ a polynomial of degree $3 p+1$.

Corollary 2.8 is proved by induction, using the inductive hypothesis on $\Phi_{t}$ and diagonalising $\mathcal{L}^{*}$ as in (2.16). The time estimate of Corollary 2.9 is proved by using $(2.9)$ on $\partial_{t}^{p} \Phi$. The space estimate of Corollary 2.9 is proved by diagonalising $\mathcal{L}^{*}$ as in (2.16) and induction: first by using 2.9 on $\Phi_{t}$, and then by applying the inductive hypothesis on $\Phi_{t}$. Indeed, we have the following continuous inclusions:

$$
\begin{aligned}
H^{p}\left(0, T ; \mathbf{H}^{-1}(0, L)\right) \cap L^{2}(0, T & \left.; \mathbf{H}^{3 p-2}(0, L)\right) \\
& \subset H^{1}\left(0, T ; \mathbf{H}^{3(p-1)-2 \frac{p-1}{p}-\frac{1}{p}}(0, L)\right) \subset H^{1}\left(0, T ; \mathbf{H}^{3(p-1)-2}(0, L)\right) .
\end{aligned}
$$

Remark 2.10. We have for 1.7 regularity results analogous to those in Proposition 2.6, Corollary 2.8 and Corollary 2.9. 


\section{A Carleman inequality for the solutions of (1.8)}

In order to shorten the notation, we define for a function $g \in C([0, L])$ and any subset $X \subset$ $[0, L]$ the integral:

$$
\int_{X} g:=\sum_{x \in X} g(x)
$$

The structure of this section is the following: first we present the weights, and secondly we state and prove the Carleman inequality.

\subsection{Definition of the weights and an auxiliary result}

Let $L>0$ and $\omega \subset(0, L)$ be an open subdomain. We consider $\eta \in C^{3}([0, L])$ a function satisfying:

$$
\|\eta\|_{\infty}=1, \quad \inf _{(0, L) \backslash \omega}\left|\eta^{\prime}\right|>0, \quad \sup _{(0, L)} \eta^{\prime \prime}<0, \quad \eta(0)=\eta(L)=0 .
$$

With that auxiliary function we define the following classical weights:

$$
\begin{aligned}
\alpha(t, x):=\frac{e^{\lambda(2 m+2)}-e^{\lambda(2 m+\eta(x))}}{t^{m}(T-t)^{m}}, \quad \xi(t, x):=\frac{e^{\lambda(2 m+\eta(x))}}{t^{m}(T-t)^{m}}, \\
\alpha^{*}(t):=\sup _{x \in(0, L)} \alpha(t, x), \quad \xi^{*}(t):=\inf _{x \in(0, L)} \xi(t, x),
\end{aligned}
$$

for $m \in \mathbb{R}^{+}$and $\lambda>0$ to be fixed later. Similar weights were first defined in [25], but this version is taken from [23. We remark that $(3.1)_{3}$ and $(3.1)_{4}$ imply the equalities:

$$
\alpha^{*}(t)=\alpha(t, 0)=\alpha(t, L), \quad \xi^{*}(t)=\xi(t, 0)=\xi(t, L) .
$$

We recall that for all $i \in \mathbb{N}_{*}$ there is $C>0$ such that if $\lambda \geq C$ we have the estimate:

$$
\left|\partial_{t}^{i} \xi\right|+\left|\partial_{t}^{i} \alpha\right| \leq C T^{i}\left(\xi^{*}\right)^{1+i / m}
$$

Next, we define the weights:

$$
\beta(t, x):=\frac{e^{\lambda(2 m+2)}-e^{\lambda(2 m+\eta(x))}}{\left(T^{2} l(t / T)\right)^{m}}, \quad \beta^{*}(t):=\sup _{x \in(0, L)} \beta(t, x),
$$

for $l(t) \in C^{\infty}([0,1])$ a positive function satisfying:

$$
l(t)=t(1-t), \text { in }[3 / 4,1], \quad l(t)>t(1-t), \text { in }[0,3 / 4), \quad l^{\prime}(t)<0, \text { in }[0,1] .
$$

An easy consequence of 3.6 is that

$$
\beta^{*} \leq \alpha^{*} \text { in }[0, T] .
$$


As for these weights, we have the following bound for $\lambda \geq C$ :

$$
\left|\beta_{t}^{*}\right|=\left|m T l^{\prime}(t / T) \frac{e^{\lambda(2 m+2)}-e^{2 m \lambda}}{\left(T^{2} l(t / T)\right)^{m+1}}\right| \leq C T\left(\beta^{*}\right)^{1+1 / m} .
$$

In general, for all $i \in \mathbb{N}_{*}$ there is $C>0$ such that if $\lambda \geq C$ we have the estimates:

$$
\left|\partial_{t}^{i} \beta^{*}\right| \leq C T^{i}\left(\beta^{*}\right)^{1+i / m}, \quad\left|\partial_{t}^{i} \beta^{*}\right| \leq C T^{i}\left(\xi^{*}\right)^{1+i / m} .
$$

Finally, for all $\delta>0$ and $k \in \mathbb{R}^{+}$there is $C>0$ such that for all $s \in \mathbb{R}^{+}$:

$$
\left(s \beta^{*}\right)^{k} \leq C e^{\delta s \beta^{*}} .
$$

Indeed, $x^{k} e^{-\delta x} \in L^{\infty}\left(\mathbb{R}^{+}, d x\right)$.

In order to prove a Carleman inequality for (1.8) we need the following technical result:

Proposition 3.1. Let $L>0, i \geq 0, j, k_{1}, k_{2} \in \mathbb{R}, k_{3} \in \mathbb{R} \backslash\{0\}, m \geq 1 / 2$ and $\omega \subset(0, L)$. There is $C>0$ such that for all $\lambda \geq C, s \geq C\left(T^{m}+T^{2 m}\right)$, and $w \in H^{2,3}(Q)$ we have the estimate

$$
\begin{array}{r}
s^{1+i} \lambda^{2+j} \iint_{Q} e^{-2 s \alpha} \xi^{1+i}\left|w_{x x}\right|^{2}+s^{3+i} \lambda^{4+j} \iint_{Q} e^{-2 s \alpha} \xi^{3+i}\left|w_{x}\right|^{2}+s^{5+i} \lambda^{6+j} \iint_{Q} e^{-2 s \alpha} \xi^{5+i} w^{2} \\
\leq C\left(s^{i} \lambda^{j} \iint_{Q} e^{-2 s \alpha} \xi^{i} \tilde{g}^{2}+s^{1+i} \lambda^{2+j} \iint_{Q_{\omega}} e^{-2 s \alpha} \xi^{1+i}\left|w_{x x}\right|^{2}+s^{5+i} \lambda^{6+j} \iint_{Q_{\omega}} e^{-2 s \alpha} \xi^{5+i} w^{2}\right. \\
\left.+s^{-1+i} \lambda^{-1+j} \int_{0}^{T} \int_{\{0, L\}} e^{-2 s \alpha} \xi^{-1+i}\left|w_{t}\right|^{2}\right),
\end{array}
$$

for

$$
\tilde{g}:=w_{t}+k_{1} w_{x}+k_{2} w_{x x}+k_{3} w_{x x x} .
$$

Proposition 3.1 can be proved for $i=j=k_{1}=k_{2}=0$ by following, step by step, with some small changes the proof presented in [11, Proposition 3.1] and in [5, Theorem 3.1] (which are based on the proof presented in [25] for the heat equation). In addition, we have for any $i \geq 0$ that $s^{i / 2} e^{-s \alpha} \xi^{i / 2} w \in H^{2,3}(Q)$, so we just have to replace $w$ by $s^{i / 2} \xi^{i / 2} w$ in (3.11) and do the usual absorptions. As for $j$, it suffices to multiply (3.11) by $\lambda^{j}$ at both sides of the inequality. Finally, we can prove it for any $k_{1}, k_{2} \in \mathbb{R}$ by considering that $w_{t}+k_{3} w_{x x x}=\tilde{g}-k_{1} w_{x}-k_{2} w_{x x}$, and then absorbing the first and second order term with the left-hand side of (3.11).

\subsection{The Carleman estimate}

In this section we prove the following Carleman estimate: 
Proposition 3.2. Let $L>0, \omega \subset(0, L)$ be an open subset, $m \geq 12$ and assume that (1.4) is satisfied. There is $C>0$ such that for all $\lambda \geq C, s \geq C\left(T^{m}+T^{3 m}\right), \Phi^{T} \in \mathbf{L}^{2}(0, L)$ and $G \in \mathbf{L}^{2}(Q)$ we have the estimate

$$
s^{6} \lambda^{6} \int_{0}^{T} e^{-2 s \alpha^{*}-10 s \beta^{*}}\left(\xi^{*}\right)^{6}\|\Phi\|_{\mathbf{H}^{2}(0, L)}^{2} \leq C\left(\iint_{Q_{\omega}} e^{-s \alpha^{*}-10 s \beta^{*}} \phi^{2}+\iint_{Q} e^{-8 s \beta^{*}}|G|^{2}\right),
$$

for $\Phi$ the energy solution of 1.8 .

Remark 3.3. We have that $s \geq C\left(T^{m}+T^{3 m}\right)$ implies that $s \geq C T^{2 m}$, which implies that $s \xi^{*} \geq 1$.

The structure of the proof is the following: first we reduce it to the case in which the solution is regular, second we decompose $\Phi$ as the sum of the solutions of three Cauchy problems, third we get regularity estimates for the first two, fourth we deduce a PDE that just contains the second component of the third auxiliary variable, fifth we get a Carleman estimate for the third auxiliary variable, sixth we estimate the trace terms, seventh we estimate the local terms, and finally we deduce a Carleman estimate just in terms of a local term of $\phi$.

Remark 3.4. As explained in the introduction, the proof of Proposition 3.2 presents the novelty that we decompose $\Phi$ as the sum of the solutions of three Cauchy problems to overcome the fact that the adjoint variable is not regular enough. The main difference in considering three Cauchy problems instead of two (as it has been done in the literature) is that in many parts of the proof we need to use the weights $(3.5)$ instead of $(3.2)$ as a way to ensure that the solutions of those Cauchy problems belong to $\mathbf{L}^{2}(Q)$. In addition, there is a second novelty which is to use a factorization to simplify the sixth order differential equation that we get when we uncouple.

Proof of Proposition 3.2. Step 1: reducing to regular solutions. By linearity arguments, density arguments and Lemma 2.5 it suffices to prove (3.13) for $G \in \mathcal{D}(Q)$ and $\Phi^{T} \in \mathcal{D}(0, L)$. In those cases we have by Lemma 2.5 that $\Phi \in \mathbf{C}^{\infty}(\bar{Q})$. Thus, from now we suppose that $G \in \mathcal{D}(Q)$ and $\Phi^{T} \in \mathcal{D}(0, L)$.

Step 2: Decomposition of $\Phi$. We consider that

$$
e^{-5 s \beta^{*}} \Phi=\hat{\Phi}+\bar{\Phi}+\tilde{\Phi}
$$

for $\hat{\Phi}:=(\hat{\varphi}, \hat{\phi})$ the solution of the system

$$
\begin{cases}\mathcal{L}^{*} \hat{\Phi}=e^{-5 s \beta^{*}} G, & \text { in } Q, \\ \hat{\Phi}(\cdot, 0)=\hat{\Phi}(\cdot, L)=\hat{\Phi}_{x}(\cdot, 0)=0, & \text { on }(0, T), \\ \hat{\Phi}(T, \cdot)=0, & \text { on }(0, L),\end{cases}
$$


$\bar{\Phi}:=(\bar{\varphi}, \bar{\phi})$ the solution of the system

$$
\begin{cases}\mathcal{L}^{*} \bar{\Phi}=5 s \beta_{t}^{*} \hat{\Phi}, & \text { in } Q, \\ \bar{\Phi}(\cdot, 0)=\bar{\Phi}(\cdot, L)=\bar{\Phi}_{x}(\cdot, 0)=0, & \text { on }(0, T), \\ \bar{\Phi}(T, \cdot)=0, & \text { on }(0, L),\end{cases}
$$

and $\tilde{\Phi}:=(\tilde{\varphi}, \tilde{\phi})$ the solution of the system

$$
\begin{cases}\mathcal{L}^{*} \tilde{\Phi}=5 s \beta_{t}^{*}\left(e^{-5 s \beta^{*}} \Phi-\hat{\Phi}\right), & \text { in } Q, \\ \tilde{\Phi}(\cdot, 0)=\tilde{\Phi}(\cdot, L)=\tilde{\Phi}_{x}(\cdot, 0)=0, & \text { on }(0, T), \\ \tilde{\Phi}(T, \cdot)=0, & \text { on }(0, L) .\end{cases}
$$

We remark that because of (3.14), $\tilde{\Phi}$ satisfies:

$$
\begin{cases}\mathcal{L}^{*} \tilde{\Phi}=5 s \beta_{t}^{*}(\bar{\Phi}+\tilde{\Phi}), & \text { in } Q, \\ \tilde{\Phi}(\cdot, 0)=\tilde{\Phi}(\cdot, L)=\tilde{\Phi}_{x}(\cdot, 0)=0, & \text { on }(0, T), \\ \tilde{\Phi}(T, \cdot)=0, & \text { on }(0, L) .\end{cases}
$$

Moreover, from Proposition 2.6, Corollary 2.8 and Corollary 2.9 we deduce that

$$
\hat{\Phi}, \bar{\Phi}, \tilde{\Phi} \in \mathbf{C}^{\infty}(\bar{Q})
$$

Step 3: estimates of the solutions of (3.15) and (3.16). First of all, we obtain from 2.10 that:

$$
\|\hat{\Phi}\|_{L^{2}\left(0, T ; \mathbf{H}^{2}(0, L)\right)} \leq q(T)\left\|e^{-5 s \beta^{*}} G\right\|_{\mathbf{L}^{2}(Q)} .
$$

We recall that $q$ stands for a generic polynomial of positive coefficient that might be different each time it appears.

Moreover, using (2.9) for system (3.15) and $(3.6)_{3}$, we have that for all $\delta>0$ there is a polynomial $q$ of positive coefficients such that, for all $t \in[0, T]$,

$$
\|\hat{\Phi}(t, \cdot)\|_{\mathbf{L}^{2}(0, L)} \leq q(T-t)\left\|e^{-5 s \beta^{*}} G\right\|_{\mathbf{L}^{2}((t, T) \times(0, L))} \leq q(T) e^{-\delta s \beta^{*}(t)}\left\|e^{-(5-\delta) s \beta^{*}} G\right\|_{\mathbf{L}^{2}(Q)} .
$$

This allows us to estimate $\left\|s \beta_{t t}^{*} \hat{\Phi}\right\|_{\mathbf{L}^{2}(Q)}$ and $\left\|\left(s \beta_{t}^{*}\right)^{2} \hat{\Phi}\right\|_{\mathbf{L}^{2}(Q)}$ in terms of $\left\|e^{-(5-\delta) s \beta^{*}} G\right\|_{\mathbf{L}^{2}(Q)}$. Consequently, looking at the system satisfied by $\beta_{t}^{*} \hat{\Phi}$, from $\left.2.10,(3.9)_{1}, 3.10\right)$ and 3.20 we get

$$
\left\|s \beta_{t}^{*} \hat{\Phi}\right\|_{L^{2}\left(0, T ; \mathbf{H}^{2}(0, L)\right) \cap H^{1}\left(0, T ; \mathbf{H}^{-1}(0, L)\right)} \leq q(T)\left\|e^{-(5-\delta) s \beta^{*}} G\right\|_{\mathbf{L}^{2}(Q)} .
$$

Thus, using (2.26) for $p=1$, from (3.21) if follows that

$$
\|\bar{\Phi}\|_{H^{1}\left(0, T ; \mathbf{H}^{1}(0, L)\right) \cap L^{2}\left(0, T ; \mathbf{H}^{4}(0, L)\right)} \leq q(T)\left\|e^{-(5-\delta) s \beta^{*}} G\right\|_{\mathbf{L}^{2}(Q)} .
$$


Finally, since $\lambda \geq C$ and $s \geq C\left(T^{m}+T^{3 m}\right)$, from 3.10$)$ and $(3.6)_{3}$ we obtain the estimate

$$
T \leq C \frac{s^{1 / m}}{T^{2}} \leq C\left(s \beta^{*}(0)\right)^{1 / m} \leq C e^{s \delta \beta^{*}(0)} \leq C e^{s \delta \beta^{*}(t)}, \quad \forall t \in[0, T] .
$$

Consequently, taking $\delta>0$ small enough and keeping the interesting terms, for $\lambda \geq C$ and $s \geq C\left(T^{m}+T^{3 m}\right)$ we have

$$
\|\hat{\Phi}\|_{L^{2}\left(0, T ; \mathbf{H}^{2}(0, L)\right)}+\|\bar{\Phi}\|_{H^{1}\left(0, T ; \mathbf{H}^{1}(0, L)\right) \cap L^{2}\left(0, T ; \mathbf{H}^{4}(0, L)\right)} \leq C\left\|e^{-4 s \beta^{*}} G\right\|_{\mathbf{L}^{2}(Q)} .
$$

Step 4: uncoupling (3.18). We now get an equation containing just $\tilde{\phi}$. By applying the operator $-\partial_{t}-\partial_{x}^{3}$ to the second equation of $(3.18)_{1}$ and using the first equation of $(3.18)_{1}$, we obtain the PDE:

$$
\begin{aligned}
c \tilde{\phi}_{t t}+c \tilde{\phi}_{t x x x}+r \tilde{\phi}_{t x}+r \partial_{x}^{4} \tilde{\phi}-a^{2} b \partial_{x}^{6} \tilde{\phi}+\tilde{\phi}_{t x x x} & +\partial_{x}^{6} \tilde{\phi} \\
& =5 a s \beta_{t}^{*} \partial_{x}^{3}(\bar{\varphi}+\tilde{\varphi})-5 s\left(\partial_{t}+\partial_{x}^{3}\right)\left(\beta_{t}^{*}(\bar{\phi}+\tilde{\phi})\right) .
\end{aligned}
$$

With the objective of simplifying the left-hand side of (3.25), we show the existence of $\kappa_{1}, \kappa_{2}, \tilde{\kappa}_{1}, \tilde{\kappa}_{2} \in \mathbb{R}$ such that the following equality is satisfied:

$$
\begin{aligned}
c\left(\partial_{t}+\tilde{\kappa}_{1} \partial_{x}+\kappa_{1} \partial_{x}^{3}\right)\left(\partial_{t}+\tilde{\kappa}_{2} \partial_{x}+\kappa_{2} \partial_{x}^{3}\right) \tilde{\phi} & \\
& =\tilde{\kappa}_{1} \tilde{\kappa}_{2} \partial_{x}^{2} \tilde{\phi}-5 s \beta_{t}^{*} \partial_{x}^{3}(\bar{\varphi}+\tilde{\varphi})-5 s\left(\partial_{t}+\partial_{x}^{3}\right)\left(\beta_{t}^{*}(\bar{\phi}+\tilde{\phi})\right) .
\end{aligned}
$$

The higher order terms form the system:

$$
\left\{\begin{array}{l}
\kappa_{1}+\kappa_{2}=1+\frac{1}{c} \\
\kappa_{1} \kappa_{2}=\frac{1-a^{2} b}{c} .
\end{array}\right.
$$

Thus, we search for $\kappa_{1}$ such that:

$$
\kappa_{1}^{2}-\frac{c+1}{c} \kappa_{1}+\frac{1-a^{2} b}{c}=0 .
$$

The discriminant of (3.28) is given by:

$$
\frac{(c+1)^{2}}{c^{2}}-4 \frac{1-a^{2} b}{c}=\frac{c^{2}+2 c+1-4 c+4 a^{2} b c}{c^{2}}=\frac{(c-1)^{2}+4 a^{2} b c}{c^{2}}>0 ;
$$

hence, equation (3.28) and system (3.27) have two real solutions. As for the coefficients of the terms of order 4 , we find the system

$$
\left\{\begin{array}{l}
\tilde{\kappa}_{1}+\tilde{\kappa}_{2}=\frac{r}{c}, \\
\kappa_{2} \tilde{\kappa}_{1}+\kappa_{1} \tilde{\kappa}_{2}=\frac{r}{c},
\end{array}\right.
$$

which clearly has at least a real solution $\left(\tilde{\kappa}_{1}, \tilde{\kappa}_{2}\right)$. Consequently, the decomposition $(3.26)$ is possible. In particular, we define the auxiliary variable

$$
\theta:=\left(\partial_{t}+\tilde{\kappa}_{2} \partial_{x}+\kappa_{2} \partial_{x}^{3}\right) \tilde{\phi} .
$$


Step 5: a Carleman estimate for $\tilde{\Phi}$. We have the following Carleman estimates:

- If we use Proposition 3.1 on $\tilde{\phi}$ for $\left(k_{1}, k_{2}, k_{3}\right)=\left(\tilde{\kappa}_{2}, 0, \kappa_{2}\right)$ and consider the homogeneous Dirichlet boundary conditions, we obtain that:

$$
\begin{aligned}
& s^{6} \lambda^{7} \iint_{Q} e^{-2 s \alpha} \xi^{6}\left|\tilde{\phi}_{x x}\right|^{2}+s^{8} \lambda^{9} \iint_{Q} e^{-2 s \alpha} \xi^{8}\left|\tilde{\phi}_{x}\right|^{2}+s^{10} \lambda^{11} \iint_{Q} e^{-2 s \alpha} \xi^{10} \tilde{\phi}^{2} \\
& \quad \leq C\left(s^{5} \lambda^{5} \iint_{Q} e^{-2 s \alpha} \xi^{5} \theta^{2}+s^{6} \lambda^{7} \iint_{Q_{\omega}} e^{-2 s \alpha} \xi^{6}\left|\tilde{\phi}_{x x}\right|^{2}+s^{10} \lambda^{11} \iint_{Q_{\omega}} e^{-2 s \alpha} \xi^{10} \tilde{\phi}^{2}\right) .
\end{aligned}
$$

- If we use Proposition 3.1 on $\tilde{\phi}_{x}$ and recall the boundary conditions of $\tilde{\Phi}$, we get:

$$
\begin{array}{r}
s^{4} \lambda^{5} \iint_{Q} e^{-2 s \alpha} \xi^{4}\left|\tilde{\phi}_{x x x}\right|^{2}+s^{6} \lambda^{7} \iint_{Q} e^{-2 s \alpha} \xi^{6}\left|\tilde{\phi}_{x x}\right|^{2}+s^{8} \lambda^{9} \iint_{Q} e^{-2 s \alpha} \xi^{8}\left|\tilde{\phi}_{x}\right|^{2} \\
\leq C\left(s^{3} \lambda^{3} \iint_{Q} e^{-2 s \alpha} \xi^{3}\left|\theta_{x}\right|^{2}+s^{4} \lambda^{5} \iint_{Q_{\omega}} e^{-2 s \alpha} \xi^{2}\left|\tilde{\phi}_{x x x}\right|^{2}+s^{8} \lambda^{9} \iint_{Q_{\omega}} e^{-2 s \alpha} \xi^{8}\left|\tilde{\phi}_{x}\right|^{2}\right. \\
\left.+s^{2} \lambda^{2} \int_{0}^{T} \int_{\{L\}} e^{-2 s \alpha} \xi^{2}\left|\tilde{\phi}_{t x}\right|^{2}\right) .
\end{array}
$$

- If we use Proposition 3.1 on $\theta$ for $\left(k_{1}, k_{2}, k_{3}\right)=\left(\tilde{\kappa}_{1}, 0, \kappa_{1}\right)$, we get from $c>0,3.29$ and (3.26) the following estimate:

$$
\begin{aligned}
& s^{2} \lambda^{3} \iint_{Q} e^{-2 s \alpha} \xi^{2}\left|\theta_{x x}\right|^{2}+s^{4} \lambda^{5} \iint_{Q} e^{-2 s \alpha} \xi^{4}\left|\theta_{x}\right|^{2}+s^{6} \lambda^{7} \iint_{Q} e^{-2 s \alpha} \xi^{6} \theta^{2} \\
& \leq C\left(s \lambda \iint_{Q} e^{-2 s \alpha} \xi\left[\tilde{\kappa}_{1} \tilde{\kappa}_{2} \tilde{\phi}_{x x}-5 s \beta_{t}^{*}\left(\bar{\varphi}_{x x x}+\tilde{\varphi}_{x x x}\right)-5 s\left(\partial_{t}+\partial_{x}^{3}\right)\left(\beta_{t}^{*}(\bar{\phi}+\tilde{\phi})\right)\right]^{2}\right. \\
& \left.\quad+s^{2} \lambda^{3} \iint_{Q_{\omega}} e^{-2 s \alpha} \xi^{2}\left|\theta_{x x}\right|^{2}+s^{6} \lambda^{7} \iint_{Q_{\omega}} e^{-2 s \alpha} \xi^{6} \theta^{2}+\int_{0}^{T} \int_{\{0, L\}} e^{-2 s \alpha}\left|\theta_{t}\right|^{2}\right) .
\end{aligned}
$$

- From 3.29 we have:

$$
s^{4} \lambda^{5} \iint_{Q} e^{-2 s \alpha} \xi^{4}\left|\tilde{\phi}_{t}\right|^{2} \leq C s^{4} \lambda^{5} \iint_{Q} e^{-2 s \alpha} \xi^{4}\left(\left|\tilde{\phi}_{x x x}\right|^{2}+\left|\tilde{\phi}_{x}\right|^{2}+\theta^{2}\right) .
$$

- If we consider the second equation of $(3.18)_{1}$, it follows that:

$$
s^{4} \lambda^{5} \iint_{Q} e^{-2 s \alpha} \xi^{4}\left|\tilde{\varphi}_{x x x}\right|^{2} \leq C s^{4} \lambda^{5} \iint_{Q} e^{-2 s \alpha} \xi^{4}\left(\left|\tilde{\phi}_{x x x}\right|^{2}+\left|\tilde{\phi}_{x}\right|^{2}+\left|\tilde{\phi}_{t}\right|^{2}+s^{2}\left(\beta_{t}^{*}\right)^{2} \bar{\phi}^{2}+s^{2}\left(\beta_{t}^{*}\right)^{2} \tilde{\phi}^{2}\right) \text {. }
$$

- Thanks to the second equation of $(3.18)_{1}, 3.29$ and Poincaré inequality (Proposition 2.1 for $i=0$ ) we obtain:

$$
\begin{aligned}
s^{6} \lambda^{6} \int_{0}^{T} & e^{-2 s \alpha^{*}}\left(\xi^{*}\right)^{6}\|\tilde{\varphi}\|_{H^{2}(0, L)}^{2} \\
& \leq C s^{6} \lambda^{6} \iint_{Q} e^{-2 s \alpha}\left(\xi^{*}\right)^{6}\left(\left|\tilde{\phi}_{x x}\right|^{2}+\left|\tilde{\phi}_{x}\right|^{2}+\tilde{\phi}^{2}+\theta^{2}+s^{2}\left(\beta_{t}^{*}\right)^{2} \bar{\phi}^{2}+s^{2}\left(\beta_{t}^{*}\right)^{2} \tilde{\phi}^{2}\right) .
\end{aligned}
$$


- Considering the first equation of $(3.18)_{1}$ the following holds:

$$
\begin{aligned}
& s^{7 / 2} \lambda^{4} \iint_{Q} e^{-2 s \alpha^{*}}\left(\xi^{*}\right)^{7 / 2}\left|\tilde{\varphi}_{t}\right|^{2} \\
& \quad \leq C s^{7 / 2} \lambda^{4} \iint_{Q} e^{-2 s \alpha^{*}}\left(\left(\xi^{*}\right)^{7 / 2}\left(\left|\tilde{\varphi}_{x x x}\right|^{2}+\left|\tilde{\phi}_{x x x}\right|^{2}+\left(5 s \beta_{t}^{*}\right)^{2}\left(\tilde{\varphi}^{2}+\bar{\varphi}^{2}\right)\right) .\right.
\end{aligned}
$$

We recall that because of $(3.9)_{2}$ we have for $\lambda \geq C$ and $s \geq C\left(T^{m}+T^{3 m}\right)$ the estimate

$$
\left(s \beta_{t}^{*}\right)^{2} \leq C s^{2+2 / m}\left(\xi^{*}\right)^{2+2 / m} .
$$

Thus, the zero-order term of $\tilde{\varphi}$ in the right-hand side of (3.36) can be absorbed by the left-hand side of $(3.35)$ if $m \geq 4, \lambda \geq C$ and $s \geq C\left(T^{m}+T^{3 m}\right)$.

So, considering $(3.9)_{2}, 3.24$ and $\left.3.30-3.36\right)$, after some easy absorptions and majorations we obtain for $m \geq 4, \lambda \geq C$ and $s \geq C\left(T^{m}+T^{3 m}\right)$ that:

$$
\begin{aligned}
& s^{4} \lambda^{5} \iint_{Q} e^{-2 s \alpha} \xi^{4}\left|\tilde{\phi}_{t}\right|^{2}+\sum_{i=0}^{3} s^{10-2 i} \lambda^{11-2 i} \iint_{Q} e^{-2 s \alpha} \xi^{10-2 i}\left|\partial_{x}^{i} \tilde{\phi}\right|^{2} \\
& +s^{7 / 2} \lambda^{4} \iint_{Q} e^{-2 s \alpha^{*}}\left(\xi^{*}\right)^{7 / 2}\left|\tilde{\varphi}_{t}\right|^{2}+s^{4} \lambda^{5} \iint_{Q} e^{-2 s \alpha} \xi^{4}\left|\tilde{\varphi}_{x x x}\right|^{2}+s^{6} \lambda^{6} \int_{0}^{T} e^{-2 s \alpha^{*}}\left(\xi^{*}\right)^{6}\|\tilde{\varphi}\|_{H^{2}(0, L)}^{2} \\
& +\sum_{i=0}^{2} s^{6-2 i} \lambda^{7-2 i} \iint_{Q} e^{-2 s \alpha} \xi^{6-2 i}\left|\partial_{x}^{i} \theta\right|^{2}+\|\hat{\Phi}\|_{L^{2}\left(0, T ; \mathbf{H}^{2}(0, L)\right)}^{2}+\|\bar{\Phi}\|_{H^{1}\left(0, T ; \mathbf{H}^{1}(0, L)\right) \cap L^{2}\left(0, T ; \mathbf{H}^{4}(0, L)\right)}^{2} \\
& \leq C\left(\sum_{i=0}^{3} s^{10-2 i} \lambda^{11-2 i} \iint_{Q_{\omega}} e^{-2 s \alpha} \xi^{10-2 i}\left|\partial_{x}^{i} \tilde{\phi}\right|^{2}+\sum_{i=0,2} s^{6-2 i} \lambda^{7-2 i} \iint_{Q_{\omega}} e^{-2 s \alpha} \xi^{6-2 i}\left|\partial_{x}^{i} \theta\right|^{2}\right. \\
& \left.+\int_{0}^{T} \int_{\{0, L\}} e^{-2 s \alpha}\left|\theta_{t}\right|^{2}+s^{2} \lambda^{2} \int_{0}^{T} \int_{\{L\}} e^{-2 s \alpha} \xi^{2}\left|\tilde{\phi}_{t x}\right|^{2}+\iint_{Q} e^{-8 s \beta^{*}}|G|^{2}\right)
\end{aligned}
$$

Step 6: absorption of the trace terms. Let us first show how to absorb the trace term of $\phi$. We recall (3.3). From trace interpolation estimates and Young inequality we get:

$$
\begin{aligned}
s^{2} \lambda^{2} \int_{0}^{T} \int_{\{L\}} e^{-2 s \alpha} \xi^{2}\left|\tilde{\phi}_{t x}\right|^{2} \leq C s^{2} \lambda^{2} \int_{0}^{T} e^{-2 s \alpha \star}\left(\xi^{*}\right)^{2}\left\|\tilde{\phi}_{t}\right\|_{H^{2}(0, L)}^{3 / 2}\left\|\tilde{\phi}_{t}\right\|_{L^{2}(0, L)}^{1 / 2} \\
\quad \leq C\left(s^{4} \lambda^{4} \iint_{Q} e^{-2 s \alpha^{*}}\left(\xi^{*}\right)^{4}\left|\tilde{\phi}_{t}\right|^{2}+s^{4 / 3} \lambda^{4 / 3} \int_{0}^{T} e^{-2 s \alpha^{*}}\left(\xi^{*}\right)^{4 / 3}\left\|\tilde{\phi}_{t}\right\|_{H^{2}(0, L)}^{2}\right) .
\end{aligned}
$$

It is clear that the first term in the right-hand side of (3.38) can be absorbed by the left-hand side of (3.37) for $\lambda \geq C$. As for the second term, we deduce that:

$$
\begin{aligned}
& s^{4 / 3} \lambda^{4 / 3} \int_{0}^{T} e^{-2 s \alpha^{*}}\left(\xi^{*}\right)^{4 / 3}\left\|\tilde{\phi}_{t}\right\|_{H^{2}(0, L)}^{2} \\
& \quad \leq C \int_{0}^{T}\left|\left(s^{2 / 3} \lambda^{2 / 3} e^{-s \alpha^{*}}\left(\xi^{*}\right)^{2 / 3}\right)_{t}\right|^{2}\|\tilde{\phi}\|_{H^{2}(0, L)}^{2}+\left\|s^{2 / 3} \lambda^{2 / 3} e^{-s \alpha^{*}}\left(\xi^{*}\right)^{2 / 3} \tilde{\phi}\right\|_{H^{1}\left(0, T ; H^{2}(0, L)\right)}^{2} .
\end{aligned}
$$


It can be proved easily that the first term in the right-hand side of (3.39) can be absorbed by the left-hand side of (3.37) using that $m \geq 4$. As for the second one, using (2.10) on $\Phi_{t}$ we have:

$$
\begin{aligned}
\left\|s^{2 / 3} \lambda^{2 / 3} e^{-s \alpha^{*}}\left(\xi^{*}\right)^{2 / 3} \tilde{\phi}\right\|_{H^{1}\left(0, T ; H^{2}(0, L)\right)}^{2} \leq\left(1+T^{2}\right)\left(\left\|\left(s^{2 / 3} \lambda^{2 / 3} e^{-s \alpha^{*}}\left(\xi^{*}\right)^{2 / 3}\right)_{t} \tilde{\Phi}\right\|_{\mathbf{H}^{1,0}(Q)}^{2}\right. \\
\left.+\left\|s^{2 / 3} \lambda^{2 / 3} e^{-s \alpha^{*}}\left(\xi^{*}\right)^{2 / 3} s \beta_{t}^{*} \tilde{\Phi}\right\|_{\mathbf{H}^{1,0}(Q)}^{2}+\left\|s^{2 / 3} \lambda^{2 / 3} e^{-s \alpha^{*}}\left(\xi^{*}\right)^{2 / 3} s \beta_{t}^{*} \bar{\Phi}\right\|_{\mathbf{H}^{1,0}(Q)}^{2}\right) .
\end{aligned}
$$

The third term in the right-hand side of $(3.40)$ can be estimated by $(3.24)$. Moreover, the first two terms in the right-hand side of $(3.40)$ can be absorbed by the left-hand side of (3.37) if $m \geq 12$. Indeed, the most problematic term is

$$
s^{10 / 3} \lambda^{4 / 3} \iint_{Q} e^{-2 s \alpha^{*}}\left(\left|\alpha_{t}^{*}\right|^{2}+\left|\beta_{t}^{*}\right|^{2}\right)\left(\xi^{*}\right)^{4 / 3}\left|\tilde{\Phi}_{t}\right|^{2},
$$

which can be absorbed by the left-hand side of (3.37) by considering $(3.4),(3.9)_{2}$ and that $C T^{2+2}=C T^{4} \leq s^{2 / m}$. The other terms can be estimated similarly.

Let us now estimate the trace term of $\theta$. Because of 3.29 and the Dirichlet boundary conditions, we have that:

$$
\theta_{t}=\tilde{\kappa}_{2} \tilde{\phi}_{t x}+\kappa_{2} \tilde{\phi}_{t x x x}, \quad \text { on }\{0, L\} \times(0, T) .
$$

In addition, considering that the source term in (3.17) satisfies Dirichlet boundary conditions and that $\mathcal{L}^{*}$ can be diagonalize as in $(2.16)$, we obtain from $(3.18)$ that there is $\kappa_{3} \in \mathbb{R}$ such that

$$
\theta_{t}(\cdot, 0)=0, \text { on }(0, T), \quad \theta_{t}(\cdot, L)=\kappa_{3} \tilde{\phi}_{t x}(\cdot, L), \text { on }(0, T) .
$$

Thus, by Remark 3.3 we obtain

$$
\int_{0}^{T} \int_{\{0, L\}} e^{-2 s \alpha}\left|\theta_{t}\right|^{2} \leq C s^{2} \lambda^{2} \int_{0}^{T} \int_{\{L\}} e^{-2 s \alpha} \xi^{2}\left|\tilde{\phi}_{t x}\right|^{2},
$$

a term which has already been treated.

Summing up, after the operations on this step and keeping the interesting terms in (3.37) we get if $m \geq 12, \lambda \geq C$ and $s \geq C\left(T^{m}+T^{3 m}\right)$ the following estimate:

$$
\begin{gathered}
s^{4} \lambda^{5} \iint_{Q} e^{-2 s \alpha} \xi^{4}\left|\tilde{\phi}_{t}\right|^{2}+\sum_{i=0}^{3} s^{10-2 i} \lambda^{11-2 i} \iint_{Q} e^{-2 s \alpha} \xi^{10-2 i}\left|\partial_{x}^{i} \tilde{\phi}\right|^{2} \\
+s^{7 / 2} \lambda^{4} \iint_{Q} e^{-2 s \alpha^{*}}\left(\xi^{*}\right)^{7 / 2}\left|\tilde{\varphi}_{t}\right|^{2}+s^{4} \lambda^{5} \iint_{Q} e^{-2 s \alpha} \xi^{4}\left|\tilde{\varphi}_{x x x}\right|^{2}+s^{6} \lambda^{6} \int_{0}^{T} e^{-2 s \alpha^{*}}\left(\xi^{*}\right)^{6}\|\tilde{\varphi}\|_{H^{2}(0, L)}^{2} \\
+\|\hat{\Phi}\|_{L^{2}\left(0, T ; \mathbf{H}^{2}(0, L)\right)}^{2}+\|\bar{\Phi}\|_{H^{1}\left(0, T ; \mathbf{H}^{1}(0, L)\right) \cap L^{2}\left(0, T ; \mathbf{H}^{4}(0, L)\right)}^{2} \\
\leq C\left(\sum_{i=0}^{3} s^{10-2 i} \lambda^{11-2 i} \iint_{Q_{\omega}} e^{-2 s \alpha} \xi^{10-2 i}\left|\partial_{x}^{i} \tilde{\phi}\right|^{2}+\sum_{i=0,2} s^{6-2 i} \lambda^{7-2 i} \iint_{Q_{\omega}} e^{-2 s \alpha} \xi^{6-2 i}\left|\partial_{x}^{i} \theta\right|^{2}\right. \\
\left.+\iint_{Q} e^{-8 s \beta^{*}}|G|^{2}\right) .
\end{gathered}
$$


Step 7: absorption of the local terms. Using space interpolation and Young inequality, we obtain that:

$$
\begin{aligned}
& \sum_{i=0}^{3} s^{10-2 i} \lambda^{11-2 i} \iint_{Q_{\omega}} e^{-2 s \alpha} \xi^{10-2 i}\left|\partial_{x}^{i} \tilde{\phi}\right|^{2} \leq C \int_{0}^{T} e^{-\frac{15}{8} s \alpha^{*}}\|\tilde{\phi}\|_{H^{3}(\omega)}^{2} \\
& \quad \leq C \int_{0}^{T} e^{-\frac{15}{8} s \alpha^{*}}\|\tilde{\phi}\|_{L^{2}(\omega)}^{1 / 2}\|\tilde{\phi}\|_{H^{4}(\omega)}^{3 / 2} \leq C\left(\iint_{Q_{\omega}} e^{-s \alpha^{*}} \tilde{\phi}^{2}+\left\|e^{-\frac{13}{12} s \alpha^{*}} \tilde{\phi}\right\|_{L^{2}\left(0, T ; H^{4}(0, L)\right)}^{2}\right) .
\end{aligned}
$$

The second term on the right hand-side of $(3.42)$ can be bounded with $(2.26)$ for $p=1$ :

$$
\begin{aligned}
&\left\|e^{-\frac{13}{12} s \alpha^{*}} \tilde{\phi}\right\|_{L^{2}\left(0, T ; H^{4}(0, L)\right)}^{2} \leq C\left(\left\|\left(e^{-\frac{13}{12} s \alpha^{*}}\right)_{t} \tilde{\Phi}\right\|_{H^{1}\left(0, T ; \mathbf{H}^{-1}(0, L)\right) \cap L^{2}\left(0, T ; \mathbf{H}^{1}(0, L)\right)}^{2}\right. \\
&+\left\|s \beta_{t}^{*} e^{-\frac{13}{12} s \alpha^{*}} \tilde{\Phi}\right\|_{H^{1}\left(0, T ; \mathbf{H}^{-1}(0, L)\right) \cap L^{2}\left(0, T ; \mathbf{H}^{1}(0, L)\right)}^{2} \\
&\left.+\left\|s \beta_{t}^{*} e^{-\frac{13}{12} s \alpha^{*}} \bar{\Phi}\right\|_{H^{1}\left(0, T ; \mathbf{H}^{-1}(0, L)\right) \cap L^{2}\left(0, T ; \mathbf{H}^{1}(0, L)\right)}^{2}\right) .
\end{aligned}
$$

The third term in the right-hand side of (3.43) can be estimated with (3.24). As for the other two terms, they can be absorbed by the left-hand side of (3.41).

In order to study the local term of $\theta$, we substitute $\theta$ by the right-hand side of $(3.29)$. The spatial terms can be treated similarly as before. As for $\tilde{\phi}_{t}$ we obtain for $\lambda \geq C$ and $s \geq C\left(T^{m}+\right.$ $T^{3 m}$ ) the estimate

$$
\begin{aligned}
\sum_{i=0,2} s^{6-2 i} \lambda^{7-2 i} \iint_{Q_{\omega}} e^{-2 s \alpha} \xi^{6-2 i}\left(\partial_{x}^{i} \partial_{t} \tilde{\phi}\right)^{2} & \leq C \iint_{Q} e^{-\frac{63}{32} s \alpha^{*}}\left\|\tilde{\phi}_{t}\right\|_{L^{2}(\omega)}^{2 / 3}\left\|\tilde{\phi}_{t}\right\|_{H^{3}(\omega)}^{4 / 3} \\
& \leq C\left(\iint_{Q_{\omega}} e^{-\frac{15}{8} s \alpha^{*}} \tilde{\phi}_{t}^{2}+\int_{0}^{T} e^{-\frac{129}{64} s \alpha^{*}}\left\|\tilde{\phi}_{t}\right\|_{H^{3}(0, L)}^{2}\right) .
\end{aligned}
$$

We can treat the last term at the left hand-side of 3.44 as before thanks to interpolation results, Corollary 2.8 (for $p=2$ ), Corollary 2.9 (for $p=1$ ), 3.23) and (3.7). As for the first term in the right-hand side of (3.44), we can simply integrate by parts:

$$
\begin{aligned}
\iint_{Q_{\omega}} e^{-\frac{15}{8} s \alpha^{*}}\left|\tilde{\phi}_{t}\right|^{2}=\frac{1}{2} \iint_{Q_{\omega}}\left(e^{-\frac{15}{8} s \alpha^{*}}\right)_{t t} \tilde{\phi}^{2}-\iint_{Q_{\omega}} e^{-\frac{15}{8} s \alpha^{*}} \tilde{\phi}_{t t} \tilde{\phi} \\
\\
\quad \leq C\left(\iint_{Q_{\omega}} e^{-s \alpha^{*}} \tilde{\phi}^{2}+\iint_{Q} e^{-\frac{11}{4} s \alpha^{*}}\left|\tilde{\phi}_{t t}\right|^{2}\right) .
\end{aligned}
$$

The last term in the right-hand side of 3.45 can be estimated as before with the help of Corollary 2.8 (for $p=2$ ), Corollary 2.9 (for $p=1$ ), 3.23 and 3.7).

So, summing up and keeping the interesting terms, from 3.42 we get

$$
\begin{aligned}
s^{6} \lambda^{6} \int_{0}^{T} e^{-2 s \alpha^{*}}\left(\xi^{*}\right)^{6}\|\tilde{\Phi}\|_{\mathbf{H}^{2}(0, L)}^{2}+\|\hat{\Phi}\|_{L^{2}\left(0, T ; \mathbf{H}^{2}(0, L)\right)}^{2} & +\|\bar{\Phi}\|_{L^{2}\left(0, T ; \mathbf{H}^{2}(0, L)\right)}^{2} \\
& \leq C\left(\iint_{Q_{\omega}} e^{-s \alpha^{*}} \tilde{\phi}^{2}+\iint_{Q} e^{-8 s \beta^{*}}|G|^{2}\right) .
\end{aligned}
$$


Step 8: returning to the variable $\Phi$. Considering (3.14) and (3.24), from (3.46) we deduce that

$$
\begin{aligned}
s^{6} \lambda^{6} \int_{0}^{T} e^{-2 s \alpha^{*}}\left(\xi^{*}\right)^{6}\|\tilde{\Phi}\|_{\mathbf{H}^{2}(0, L)}^{2}+\|\hat{\Phi}\|_{L^{2}\left(0, T ; \mathbf{H}^{2}(0, L)\right)}^{2}+\|\bar{\Phi}\|_{L^{2}\left(0, T ; \mathbf{H}^{2}(0, L)\right)}^{2} & \\
& \leq C\left(\iint_{Q_{\omega}} e^{-s \alpha^{*}-10 s \beta^{*}} \phi^{2}+\iint_{Q} e^{-8 s \beta^{*}}|G|^{2}\right) .
\end{aligned}
$$

Therefore, from (3.47) and 3.14 we obtain (3.13).

Remark 3.5. The careful reader can remark that in Steps 2 and 3 of the proof of Proposition 3.2 we have just used that the dynamics of $(1.8)$ are dispersive, that we could replicate those steps if the dynamics were diffusive and that it is in steps 4 and 5 where we have used the properties

of the specific equation. In addition, the technique in step 2 for decomposing $e^{-5 s \beta^{*}} \Phi$ as the solution of three Cauchy problem can be generalized to decomposing $e^{-5 s \beta^{*}} \Phi$ as the solution of $N$ Cauchy problems, which could be useful in situations in which we need to have higher regularity for the solutions of analogous Cauchy problems. Thus, the effort of getting a Carleman estimate in dispersive or diffusive system with source term $G=0$ or $G \in \mathbf{L}^{2}(Q)$ is very similar.

\section{The nonlinear control problem}

In the following, we denote

$$
L^{r}(\rho(t)(0, T) ; V):=\left\{w: \rho(t) w \in L^{r}(0, T ; V)\right\}
$$

for $V$ a Banach space, $\rho$ a measurable function and $r \in[1,+\infty]$. Moreover, we define

$$
\|w\|_{L^{r}(\rho(t)(0, T) ; V)}:=\|\rho(t) w\|_{L^{r}(0, T ; V)} .
$$

Similarly, we define

$$
C(\rho(t)[0, T] ; V):=\{w: \rho(t) w \in C([0, T] ; V)\} .
$$

In addition, in this section we fix $(m, \lambda, s)$ so that Proposition 3.2 is satisfied and

$$
\left|s \beta_{t}^{*}\right| \leq C e^{\frac{1}{8} s \beta^{*}} .
$$

In that sense, in the following the generic constant $C$ may also depend on $s, \lambda$ and $T$.

The structure of this section is the following: first we study the controllability of the linearized problem, second we end the proof of Theorem 1.1, and thirdly we make some remarks about similar control problems which can be studied by using the technique presented in this paper. 


\subsection{Null controllability of 1.5}

Let $E$ be the couples $(U, f)$ such that:

- $U \in L^{2}\left(e^{4 s \beta^{*}}(0, T) ; \mathbf{L}^{2}(0, L)\right)$,

- $U \in L^{2}\left(e^{\frac{7}{2} s \beta^{*}}(0, T) ; \mathbf{H}_{0}^{1}(0, L)\right) \cap C\left(e^{\frac{7}{2} s \beta^{*}}[0, T] ; \mathbf{L}^{2}(0, L)\right)$,

- $f 1_{\omega} \in L^{2}\left(e^{5 s \beta^{*}}(0, T) ; L^{2}(0, L)\right)$,

- $\mathcal{L} U-\left(0, f 1_{\omega}\right) \in L^{2}\left(e^{7 s \beta^{*}}(0, T) ; \mathbf{H}^{-1}(0, L)\right)$.

Then, we have the following controllability result:

Proposition 4.1. Let $U^{0} \in \mathbf{L}^{2}(0, L), h_{1}, h_{2} \in L^{2}\left(e^{7 s \beta^{*}}(0, T) ; H^{-1}(0, L)\right)$ and assume that 1.4 is satisfied. Then, there is a solution of (1.5) satisfying $(U, f) \in E$.

Remark 4.2. If $(U, f) \in E$, we have that $U(T, \cdot)=0$.

For the proof of Proposition 4.1 we follow the approach introduced in [25] which is based on duality. In particular, we first prove the following auxiliary result:

Lemma 4.3. Let $L>0, \omega \subset(0, L)$ be an open subset and assume that (1.4) is satisfied. There is $C>0$ such that

$$
\int_{0}^{T} e^{-14 s \beta^{*}}\|\Phi\|_{\mathbf{H}_{0}^{1}(0, L)}^{2}+\left\|e^{-7 s \beta^{*}} \Phi\right\|_{C\left([0, T] ; \mathbf{L}^{2}(0, L)\right)}^{2} \leq C\left(\iint_{Q_{\omega}} e^{-10 s \beta^{*}} \phi^{2}+\iint_{Q} e^{-8 s \beta^{*}}|G|^{2}\right),
$$

for $\Phi$ the energy solution of (1.8) and $(m, \lambda, s)$ fixed in the beginning of this section.

Proof of Lemma 4.3. It is a straight consequence of $(3.5),(3.6)_{1}$, Proposition 2.6 applied to $e^{-7 s \beta^{*}} \Phi$ in $[3 T / 4, T]$ and $(3.13)$ that the following estimate holds:

$$
\int_{3 T / 4}^{T} e^{-14 s \beta^{*}}\|\Phi\|_{\mathbf{H}_{0}^{1}(0, L)}^{2}+\left\|e^{-7 s \beta^{*}} \Phi\right\|_{C\left([3 T / 4, T] ; \mathbf{L}^{2}(0, L)\right)}^{2} \leq C\left(\iint_{Q_{\omega}} e^{-10 s \beta^{*}} \phi^{2}+\iint_{Q} e^{-8 s \beta^{*}}|G|^{2}\right) .
$$

Let $\chi$ be a positive function supported in $[0,7 / 8]$ such that $\chi=1$ in $[0,3 / 4]$. By considering (3.5), the problem satisfied by $\chi(t / T) \Phi,(2.9)$ and (4.3) we easily obtain 4.2 .

Proof of Proposition 4.1. Let $P_{0}$ be the subspace of $\mathbf{C}^{3}([0, T] \times[0, L])$ of the functions $\Phi$ such that

$$
\Phi(\cdot, 0)=\Phi(\cdot, L)=\Phi_{x}(\cdot, 0)=0, \quad \text { on }[0, T] .
$$

Let $\mathfrak{a}: P_{0} \times P_{0} \rightarrow \mathbb{R}$ be the bilinear form

$$
\mathfrak{a}(\hat{\Phi}, \Phi)=\iint_{Q_{\omega}} e^{-10 s \beta^{*}} \hat{\phi} \phi+\iint_{Q} e^{-8 s \beta^{*}}\left(\mathcal{L}^{*} \hat{\Phi}\right) \cdot\left(\mathcal{L}^{*} \Phi\right)
$$


and $\ell: P_{0} \rightarrow \mathbb{R}$ the linear form

$$
\ell(\Phi)=\int_{0}^{L} U^{0} \cdot \Phi(0, x) d x+\int_{0}^{T}\left\langle\left(h_{1}, h_{2}\right), \Phi\right\rangle_{\mathbf{H}^{-1}(0, L) \times \mathbf{H}_{0}^{1}(0, L)} .
$$

Notice that the observability inequality 4.2 holds for every $\Phi \in P_{0}$ taking $G=\mathcal{L}^{*} \Phi$. Consequently, $\mathfrak{a}$ is a scalar product in $P_{0}$ and there is $C>0$ such that for all $\Phi \in P_{0}$ we have that

$$
|\ell(\Phi)| \leq C\left(\left\|e^{7 s \beta^{*}}\left(h_{1}, h_{2}\right)\right\|_{L^{2}\left(0, T ; \mathbf{H}^{-1}(0, L)\right)}+\left\|\left(u_{0}, v_{0}\right)\right\|_{\mathbf{L}^{2}(0, L)}\right) \sqrt{\mathfrak{a}(\Phi, \Phi)} .
$$

In the following we denote $P$ the completion of $P_{0}$ with respect to $\mathfrak{a}$ (and keep denoting $\mathfrak{a}$ and $\ell$ to their only continuous extensions). By Riesz theorem there is $\hat{\Phi} \in P$ such that

$$
\mathfrak{a}(\hat{\Phi}, \Phi)=\ell(\Phi), \quad \forall \Phi \in P .
$$

We define $(U, f)$ by

$$
U:=e^{-8 s \beta^{*}} \mathcal{L}^{*} \hat{\Phi}, \quad f:=-e^{-10 s \beta^{*}} \hat{\phi} 1_{\omega} .
$$

It can be proved easily that $U$ is the unique solution by transposition of

$$
\begin{cases}\mathcal{L} U=\left(h_{1}, h_{2}+f 1_{\omega}\right), & \text { in } Q, \\ U(\cdot, 0)=U(\cdot, L)=U_{x}(\cdot, L)=0, & \text { on }(0, T), \\ U(0, \cdot)=U^{0}, & \text { on }(0, L) .\end{cases}
$$

Indeed, let $\Phi$ be the solution of 1.8 for $G \in \mathcal{D}(Q)$ and for $\Phi^{T}=0$. Then, we have by Lemma 2.5 that $\Phi \in \mathbf{C}^{\infty}(\bar{Q}) \cap P_{0}$, which implies that

$$
\begin{aligned}
& \int_{0}^{T}\langle U, G\rangle_{\mathbf{H}^{-1}(0, L), \mathbf{H}_{0}^{1}(0, L)}=\iint_{Q} e^{-8 s \beta^{*}}\left(\mathcal{L}^{*} \hat{\Phi}\right) \cdot G=\iint_{Q} e^{-8 s \beta^{*}}\left(\mathcal{L}^{*} \hat{\Phi}\right) \cdot\left(\mathcal{L}^{*} \Phi\right) \\
& \quad=\mathfrak{a}(\hat{\Phi}, \Phi)-\iint_{Q_{\omega}} e^{-10 s \beta^{*}} \hat{\phi} \phi=\int_{0}^{L} U^{0} \cdot \Phi(0, x) d x+\int_{0}^{T}\left\langle\left(h_{1}, h_{2}+f 1_{\omega}\right), \Phi\right\rangle_{\mathbf{H}^{-1}(0, L) \times \mathbf{H}_{0}^{1}(0, L)}
\end{aligned}
$$

So we just have to use the density of $\mathcal{D}(Q)$ in $L^{2}\left(0, T ; \mathbf{H}_{0}^{1}(0, L)\right)$. Consequently, since $f, h_{1}, h_{2} \in$ $L^{2}\left(0, T ; H^{-1}(0, L)\right)$, we obtain from Remark 2.10 that

$$
U \in L^{2}\left(0, T ; \mathbf{H}_{0}^{1}(0, L)\right) \cap C\left([0, T] ; \mathbf{L}^{2}(0, L)\right) .
$$

As for the estimates, from 4.6 we get

$$
\iint_{Q_{\omega}} e^{10 s \beta^{*}}|f|^{2}+\iint_{Q} e^{8 s \beta^{*}}|U|^{2}<+\infty .
$$

Moreover, since $U$ is the solution by transposition of 4.7 we have that

$$
\mathcal{L} U-\left(0, f 1_{\omega}\right)=\left(h_{1}, h_{2}\right) \in L^{2}\left(e^{7 s \beta^{*}}(0, T) ; \mathbf{H}^{-1}(0, L)\right) .
$$


Finally, we consider that $\tilde{U}=e^{\frac{7}{2} s \beta^{*}} U$ satisfies:

$$
\begin{cases}\mathcal{L} \tilde{U}=\frac{7}{2} s \beta_{t}^{*} e^{\frac{7}{2} s \beta^{*}} U+e^{\frac{7}{2} s \beta^{*}}\left(h_{1}, h_{2}+f 1_{\omega}\right), & \text { in } Q, \\ \tilde{U}(\cdot, 0)=\tilde{U}(\cdot, L)=\tilde{U}_{x}(\cdot, L)=0, & \text { on }(0, T), \\ \tilde{U}(0, \cdot)=e^{\frac{7}{2} s \beta^{*}(0)} U^{0}, & \text { in }(0, L) .\end{cases}
$$

Moreover, from $U \in L^{2}\left(e^{4 s \beta^{*}}(0, T) ; \mathbf{H}^{-1}(0, L)\right)$ and 4.1 we obtain

$$
\frac{7}{2} s \beta_{t}^{*} e^{\frac{7}{2} s \beta^{*}} U+e^{\frac{7}{2} s \beta^{*}}\left(h_{1}, h_{2}+f 1_{\omega}\right) \in L^{2}\left(0, T ; \mathbf{H}^{-1}(0, L)\right) .
$$

Consequently, from Remark 2.10 we find

$$
U \in L^{2}\left(e^{\frac{7}{2} s \beta^{*}}(0, T) ; \mathbf{H}_{0}^{1}(0, L)\right) \cap C\left(e^{\frac{7}{2} s \beta^{*}}[0, T] ; \mathbf{L}^{2}(0, L)\right) .
$$

Thereby, we obtain from 4.8-4.10 that $(U, f) \in E$, which along with being the solution of 4.7) completes the proof.

\subsection{Proof of Theorem 1.1}

We now prove the local null controllability of system (1.3) by a local inversion argument. We recall that the components of $U$ are denoted by $u$ and $v$. We consider $\mathfrak{h}_{1}, \mathfrak{h}_{2}$ as in the statement of Theorem 1.1, with $C$ large enough so that $\mathfrak{h}_{1}, \mathfrak{h}_{2} \in L^{2}\left(e^{7 s \beta^{*}}(0, T) ; \mathbf{H}^{-1}(0, L)\right)$. Let $\mathcal{G}: E \rightarrow L^{2}\left(e^{7 s \beta^{*}}(0, T) ; \mathbf{H}^{-1}(0, L)\right) \times \mathbf{L}^{2}(0, L)$ be an operator defined by:

$$
\begin{aligned}
\mathcal{G}(U, f):= & \left(u_{t}+u u_{x}+u_{x x x}+a v_{x x x}+k_{1} v v_{x}+k_{2}(u v)_{x}-\mathfrak{h}_{1},\right. \\
& \left.c v_{t}+r v_{x}+v v_{x}+a b u_{x x x}+v_{x x x}+k_{2} b u u_{x}+k_{1} b(u v)_{x}-\mathfrak{h}_{2}-f 1_{\omega}, u(0, \cdot), v(0, \cdot)\right) .
\end{aligned}
$$

We recall that if $\mathcal{G}$ is $C^{1}$ and if $D \mathcal{G}[0]$ is surjective, there exists $\delta>0$ such that if $\left\|U^{0}\right\|<\delta$ there exists $(U, f) \in E$ such that

$$
\mathcal{G}(U, f)=\left(0_{\mathbb{R}^{2}}, U^{0}\right) .
$$

Hence, by Remark 4.2 we just have to check those two hypotheses to conclude the proof of Theorem 1.1:

- In order to see that $\mathcal{G}$ is $C^{1}$, since $\mathcal{G}$ is the sum of an affine form and some bilinear forms, it suffices to see that it is a bounded operator. The fact that the affine form is continuous is a straight consequence of the definition of $E$. As for the bilinear terms of (4.11), we can prove that they are bounded by considering that for all $w_{1} \in L^{\infty}\left(e^{\frac{7}{2} s \beta^{*}}(0, T) ; L^{2}(0, L)\right)$ and $w_{2} \in L^{2}\left(e^{\frac{7}{2} s \beta^{*}}(0, T) ; H_{0}^{1}(0, L)\right)$ we have the estimate

$$
\begin{aligned}
\left\|w_{1} \partial_{x} w_{2}\right\|_{L^{2}\left(e^{7 s \beta^{*}}(0, T) ; H^{-1}(0, L)\right)} \leq & C\left\|w_{1} \partial_{x} w_{2}\right\|_{L^{2}\left(e^{7 s \beta^{*}}(0, T) ; L^{1}(0, L)\right)} \\
& \leq C\left\|w_{1}\right\|_{L^{\infty}\left(e^{\frac{7}{2} s \beta^{*}}(0, T) ; L^{2}(0, L)\right)}\left\|w_{2}\right\|_{L^{2}\left(e^{\frac{7}{2} s \beta^{*}}(0, T) ; H_{0}^{1}(0, L)\right)}
\end{aligned}
$$


- As for the surjectivity of $D \mathcal{G}[0]$, we remark that

$$
D \mathcal{G}[0](U, f)=\left(\mathcal{L} U-\left(0, f 1_{\omega}\right), U(0, \cdot)\right)
$$

thus, it is a consequence of Proposition 4.1 .

\subsection{Some additional remarks}

The technique presented in this paper can be used to solve other controllability problems:

- We can prove the local null controllability of the following control system

$$
\begin{cases}u_{t}+u u_{x}+u_{x x x}+a v_{x x x}+k_{1} v v_{x}+k_{2}(u v)_{x}=\mathfrak{h}_{1}+f 1_{\omega}, & \text { in } Q, \\ c v_{t}+r v_{x}+v v_{x}+a b u_{x x x}+v_{x x x}+k_{2} b u u_{x}+k_{1} b(u v)_{x}=\mathfrak{h}_{2}, & \text { in } Q, \\ u(\cdot, 0)=u(\cdot, L)=u_{x}(\cdot, L)=0, & \text { on }(0, T), \\ v(\cdot, 0)=v(\cdot, L)=v_{x}(\cdot, L)=0, & \text { on }(0, T), \\ u(0, \cdot)=u^{0}, \quad v(0, \cdot)=v^{0}, & \text { on }(0, L),\end{cases}
$$

assuming (1.4). Indeed, we can do that by replicating the proof of Theorem 1.1 step by step.

- We can slightly improve the result presented in [16] and show that the general HirotaSatsuma system can be controlled with two forces supported in any two control domains (in [16] the authors need that one of the control domains touches the boundary). The proof is very similar to the one of Theorem 1.1, as we only have to do some minor adjustments.

- An interesting question that remains open for the control system 1.3 is the possibility of getting exact controllability results similar to those presented in [11] but with a control acting in one component; i.e. to determine if for $\tilde{L} \in(0, L)$ and $u^{*}, v^{*} \in L^{2}(0, \tilde{L})$ we can obtain $(u, v)(T, \cdot)_{\mid(0, \tilde{L})}=\left(u^{*}, v^{*}\right)$ when $\omega$ is compactly included in $(\tilde{L}, L)$.

- The second and third hypothesis in (1.4) are needed for the well-posedness of system 1.3 and its linearized system. Indeed, we use them in the proof of Proposition 2.6 to diagonalize higher order terms. In addition, these conditions have a physical meaning as explained in [26, Section 4]. Moreover, the first hypothesis in (1.4) is only necessary to prove the controllability of the linearized system (as otherwise we have two independent equations). In fact, determining the null controllability of 1.3 when $a=0$ remains an interesting open problem (in that case the other two hypotheses of 1.3 are still necessary to ensure the well-posedness of the system). Finally, there is no extra hypothesis for the coefficients which just multiply lower order terms (i.e. for $r, k_{1}$ and $k_{2}$ ) because the important terms for the well-posedness and controllability properties are the higher order ones. 


\section{References}

[1] R. A. Adams and J. J.F. Fournier. Sobolev spaces. Elsevier, 2003.

[2] F. Ammar-Khodja, A. Benabdallah, and C. Dupaix. Null-controllability of some reactiondiffusion systems with one control force. J. Math. Anal. App., 320(2):928-943, 2006.

[3] F. Ammar-Khodja, A. Benabdallah, C. Dupaix, and I. Kostin. Controllability to the trajectories of phase-field models by one control force. SIAM J. Control Optim., 42(5):1661-1680, 2003.

[4] F. Ammar-Khodja, A. Benabdallah, M. González Burgos, and L. de Teresa. Recent results on the controllability of linear coupled parabolic problems: a survey. Math. Control Relat. F., 1(3):267-306, 2011.

[5] F. D. Araruna, E. Cerpa, A. Mercado, and M. C. Santos. Internal null controllability of a linear Schrödinger-KdV system on a bounded interval. J. Differ. Equations, 260(1):653$687,2016$.

[6] J. L. Bona, G. Ponce, J.-C. Saut, and M. M. Tom. A model system for strong interaction between internal solitary waves. Comm. Math. Phys., 143(2):287-313, 1992.

[7] J. A. Bárcena-Petisco. Null controllability of a penalized Stokes problem in dimension two with one scalar control. Asymptotic Anal., 117(3-4):161-198, 2020.

[8] R. A. Capistrano-Filho, F. A. Gallego, and A. F. Pazoto. Neumann boundary controllability of the Gear-Grimshaw system with critical size restrictions on the spatial domain. Z. Angew. Math. Phys., 67(5):Art. 109, 36, 2016.

[9] R. A. Capistrano-Filho, F. A. Gallego, and A. F. Pazoto. Boundary controllability of a nonlinear coupled system of two Korteweg-de Vries equations with critical size restrictions on the spatial domain. Math. Control Signals Syst., 29(1):Art. 6, 37, 2017.

[10] R. A. Capistrano-Filho, V. Komornik, and A. F. Pazoto. Pointwise control of the linearized Gear-Grimshaw system. Evol. Equ. Control The., 9(3):693-719, 2020.

[11] R. A. Capistrano-Filho, A. F. Pazoto, and L. Rosier. Internal controllability of the Korteweg-de Vries equation on a bounded domain. ESAIM: COCV, 21(4):1076-1107, 2015.

[12] R. A. Capistrano-Filho, A. F. Pazoto, and L. Rosier. Control of a Boussinesq system of KdV-KdV type on a bounded interval. ESAIM: COCV, 25:58, 2019. 
[13] N. Carreño and S. Guerrero. Local null controllability of the $N$-dimensional Navier-Stokes system with $N-1$ scalar controls in an arbitrary control domain. J. Math. Fluid Mech., pages $1-15,2013$.

[14] N. Carreño, S. Guerrero, and M. Gueye. Insensitizing controls with two vanishing components for the three-dimensional Boussinesq system. ESAIM: COCV, 21(1):73-100, 2015.

[15] N. Carreño, E. Cerpa, and A. Mercado. Boundary controllability of a cascade system coupling fourth-and second-order parabolic equations. Syst. \& Control Lett., 133:104542, 2019.

[16] N. Carreño, E. Cerpa, and E. Crépeau. Internal null controllability of the generalized Hirota-Satsuma system. ESAIM:COCV, 26, 2020.

[17] E. Cerpa and A. F. Pazoto. A note on the paper "On the controllability of a coupled system of two Korteweg-de Vries equations". Commun. Contemp. Math., 13(1):183-189, 2011.

[18] J.-M. Coron and S. Guerrero. Null controllability of the $N$-dimensional Stokes system with N-1 scalar controls. J. Differ. Equations, 246(7):2908-2921, 2009.

[19] J.-M. Coron and P. Lissy. Local null controllability of the three-dimensional NavierStokes system with a distributed control having two vanishing components. Invent. Math., 198(3):833-880, 2014.

[20] L. De Teresa and E. Zuazua. Identification of the class of initial data for the insensitizing control of the heat equation. Commun. Pure Appl. Anal., 8(1):457-471, 2009.

[21] S. Dolecki and D. L. Russell. A general theory of observation and control. SIAM J. Control Optimization, 15(2):185-220, 1977.

[22] E. Fernández-Cara, M. González-Burgos, S. Guerrero, and J.-P. Puel. Null controllability of the heat equation with boundary Fourier conditions: the linear case. ESAIM: COCV, 12(3):442-465, 2006.

[23] E. Fernández-Cara and S. Guerrero. Global carleman inequalities for parabolic systems and applications to controllability. SIAM J. Control. and Optim., 45(4):1395-1446, 2006.

[24] E. Fernández-Cara, S. Guerrero, O. Yu. Imanuvilov, and J.-P. Puel. Some controllability results for the $N$-Dimensional Navier-Stokes and Boussinesq systems with $N-1$ scalar controls. SIAM J. Control Optim., 45(1):146-173, 2006.

[25] A. V. Fursikov and O. Yu. Imanuvilov. Controllability of evolution equations. Number 34. Seoul National University, 1996. 
[26] J. A. Gear and R. Grimshaw. Weak and strong interactions between internal solitary waves. Stud. Appl. Math., 70(3):235-258, 1984.

[27] O. Glass and S. Guerrero. Some exact controllability results for the linear KdV equation and uniform controllability in the zero-dispersion limit. Asymptotic Anal., 60(1-2):61-100, 2008 .

[28] O. Glass and S. Guerrero. Uniform controllability of a transport equation in zero diffusiondispersion limit. Math. Mod. Meth. Appl. S., 19(09):1567-1601, 2009.

[29] M. González Burgos and L. de Teresa. Controllability results for cascade systems of $m$ coupled parabolic PDEs by one control force. Port. Math., 67(1):91-113, 2010.

[30] S. Guerrero. Controllability of systems of Stokes equations with one control force: existence of insensitizing controls. Ann. I. H. Poincaré, 24:1029-1054, 2007.

[31] G. Lebeau and E. Zuazua. Null-Controllability of a System of Linear Thermoelasticity. Arch. Ration. Mech. An., 141(4):297-329, 1998.

[32] J.-L. Lions. Contrôlabilité exacte, perturbations et stabilisation de systèmes distribués. Tome 1, volume 8 of Recherches en Mathématiques Appliquées [Research in Applied Mathematics]. Masson, Paris, 1988. Contrôlabilité exacte. [Exact controllability], With appendices by E. Zuazua, C. Bardos, G. Lebeau and J. Rauch.

[33] J.-L. Lions and E. Zuazua. A generique uniqueness result for the Stokes system and its control theoretical consequences. Partial differential equations and applications: Collected Papers in Honor of Carlo Pucci, 177:221-235, 1996.

[34] S. Micu and J. H. Ortega. On the controllability of a linear coupled system of Korteweg-de Vries equations. In Mathematical and numerical aspects of wave propagation (Santiago de Compostela, 2000), pages 1020-1024. SIAM, Philadelphia, PA, 2000.

[35] S. Micu, J. H. Ortega, and A. F. Pazoto. On the controllability of a coupled system of two Korteweg-de Vries equations. Commun. Contemp. Math., 11(05):799-827, 2009.

[36] L. Rosier. Exact boundary controllability for the Korteweg-de Vries equation on a bounded domain. ESAIM: COCV, 2:33-55, 1997. 